\title{
Concise and Accessible Representations for Multidimensional Datasets: Introducing a Framework Based on the $n$ D-EVM and Kohonen Networks
}

\author{
Ricardo Pérez-Aguila and Ricardo Ruiz-Rodríguez \\ Group for Multidisciplinary Research Applied to Education and Engineering (GIMAEI), \\ The Technological University of the Mixteca (UTM), Carretera Huajuapan-Acatlima Km. 2.5, 69004 Huajuapan de León, OAX, Mexico
}

Correspondence should be addressed to Ricardo Pérez-Aguila; ricardo.perez.aguila@gmail.com

Received 24 September 2014; Revised 21 January 2015; Accepted 26 January 2015

Academic Editor: T. Warren Liao

Copyright ( 2015 R. Pérez-Aguila and R. Ruiz-Rodríguez. This is an open access article distributed under the Creative Commons Attribution License, which permits unrestricted use, distribution, and reproduction in any medium, provided the original work is properly cited.

\begin{abstract}
A new framework intended for representing and segmenting multidimensional datasets resulting in low spatial complexity requirements and with appropriate access to their contained information is described. Two steps are going to be taken in account. The first step is to specify $(n-1) \mathrm{D}$ hypervoxelizations, $n \geq 2$, as Orthogonal Polytopes whose $n$th dimension corresponds to color intensity. Then, the $n \mathrm{D}$ representation is concisely expressed via the Extreme Vertices Model in the $n$-Dimensional Space $(n \mathrm{D}$ EVM). Some examples are presented, which, under our methodology, have storing requirements minor than those demanded by their original hypervoxelizations. In the second step, 1-Dimensional Kohonen Networks (1D-KNs) are applied in order to segment datasets taking in account their geometrical and topological properties providing a non-supervised way to compact even more the proposed $n$-Dimensional representations. The application of our framework shares compression ratios, for our set of study cases, in the range 5.6496 to 32.4311 . Summarizing, the contribution combines the power of the $n \mathrm{D}$-EVM and $1 \mathrm{D}$-KNs by producing very concise datasets' representations. We argue that the new representations also provide appropriate segmentations by introducing some error functions such that our 1D-KNs classifications are compared against classifications based only in color intensities. Along the work, main properties and algorithms behind the $n \mathrm{D}$-EVM are introduced for the purpose of interrogating the final representations in such a way that it efficiently obtains useful geometrical and topological information.
\end{abstract}

\section{Introduction}

The representation of a polytope through a scheme of Hyperspatial Occupancy Enumeration is essentially a list of identical hyperspatial cells occupied by the polytope. Specific types of cells, called hypervoxels [1] are hyperboxes of a fixed size that lie in a fixed grid in the $(n-1)$-Dimensional space, $n \geq 2$. By instantiation, it is well known that a $2 \mathrm{D}$ hypervoxel is a pixel while a $3 \mathrm{D}$ hypervoxel is a voxel; the term rexel is suggested for referencing a $4 \mathrm{D}$ hypervoxel [1]. The collection of hyperboxes can be codified as an $(n-1) \mathrm{D}$ array $C_{x_{1}, x_{2}, \ldots, x_{n-1}}$. The array represents the color associated with each hypervoxel. If $C_{x_{1}, x_{2}, \ldots, x_{n-1}}=0$, the empty hypervoxel $C_{x_{1}, x_{2}, \ldots, x_{n-1}}$ represents an unoccupied region from the $(n-1) \mathrm{D}$ space. If $C_{x_{1}, x_{2}, \ldots, x_{n-1}}=c$, where $c$ is in a given color scale (black \& white, grayscale, RGB, etc.), then the occupied hypervoxel $C_{x_{1}, x_{2}, \ldots, x_{n-1}}$ represents a region from the $(n-1) \mathrm{D}$ space with intensity $c$. In fact, the set of occupied cells defines an orthogonal polytope $p$ whose vertices coincide with some of the occupied cells' vertices. By using the representation through an array, the spatial complexity of a $(n-1)$-Dimensional hypervoxelization is at least

$$
\prod_{i=1}^{n-1} n_{i}
$$

where $n_{i}, i=1,2, \ldots, n-1$, is the length of the grid along the $X_{i}$-axis. For example, a 3D grid with $n_{1}=n_{2}=n_{3}=1,000$ requires to store 1 billion $\left(1 \times 10^{9}\right)$ voxels. Moreover, according to the used color scale each voxel will have a storing requirement: if the color space is RGB then each voxel will 
require three bytes (four, if the alpha channel is considered) for codifying its corresponding intensity.

It is well known that some devices represent natively datasets through hypervoxelizations. However, sometimes their storing requirements make the manipulation and the extraction of information a difficult task. In this sense, several efforts have been made in order to reduce the spatial complexity of datasets always taking in account the information they contain, due to its importance and relevance, should be compromised as minimum as possible. For example, an algorithm for compression of datasets by means of quadtrees, in order to encode slices of data, is presented in [2]. Such encodings are used for discovering similarities between consecutive slices. In [3], 3D medical datasets are compressed via a method sustained in the use of octrees. Both works share us evidence of the spatial conciseness provided by considering the use of Solid Representation Schemes. This work is devoted to present an alternative representation for datasets whose color scales are not binary (in [4] is presented a methodology designed specifically for 3D datasets with black \& white color scale). The main idea is to specify $(n-1) \mathrm{D}$ datasets, $n \geq 2$, as $n \mathrm{D}$ polytopes where the $n$th dimension corresponds to color. Then, the $n \mathrm{D}$ representation can be concisely expressed and manipulated through a polytopes' representation scheme: the Extreme Vertices Model in the $n$-Dimensional Space $(n \mathrm{D}$ EVM). However, we take one additional step: a 1-Dimensional Kohonen Network (1D-KN) will be applied in order to segment datasets taking in account their geometry and topology providing by instance a nonsupervised intelligent way to compact even more the proposed $n \mathrm{D}$ representations. In this last sense, neural networks-based segmentation is a topic well developed in the literature. In [5] is presented the moving average self-organizing map (MA-SOM) which is applied for segmenting medical images obtained by means of $\mathrm{X}$-ray computer tomography, breast ultrasound imaging, and magnetic resonance imaging. In [6], Kohonen networks are used together with a threshold technique in order to segment satellite images. There is a review of segmenting methods based on clustering and neural networks approaches presented in [7]. In such work some experiments are presented based on the use of Multilayer Perceptrons trained with the well-known Backpropagation Algorithm. The paper by Jiang et al. [8] presents a survey of application of Neural Networks for medical support in processing images in tasks such as Image Segmentation, Edge Detection, and Computer Aided Diagnosis by means of application of the Multilayer Perceptrons, Hopfield Networks, and Self-Organizing Maps, among others. These works exemplify how the Artificial Neural Networks approach has gained confidence among Image Processing researchers due to valuable properties such as robustness to noise, plasticity, parallelism, and computational efficiency.

This work is organized as follows: Section 2 will describe the fundamentals behind the $n \mathrm{D}-\mathrm{EVM}$. The methodology for the conversion of a $(n-1) \mathrm{D}$ hypervoxelization dataset to a $n \mathrm{D}-\mathrm{EVM}$ is described in Section 3. Also, there will be presented some examples of datasets expressed under the $n \mathrm{D}$ EVM. By comparing the storing requirements between the original and the $n \mathrm{D}$-EVM representations it is established the first level of conciseness of our proposal. Section 4 describes the fundamentals behind the 1D-KNs. In Section 5 it is described the way a $1 \mathrm{D}-\mathrm{KN}$ assists us in the automatic nonsupervised segmentation of a dataset. In Section 6 how the classification provided by our $1 \mathrm{D}-\mathrm{KN}$ gives shape to the second level of conciseness by describing some examples and their final spatial requirements is presented. Section 7 provides arguments to sustain the segmentations that have been produced have good quality and they are even better than those segmentations produced by simple and direct classification based only in color intensities. For this purpose, some error functions are introduced. Finally, Section 8 presents our conclusions and lines for future research.

\section{The Extreme Vertices Model in the $n$-Dimensional Space $(n \mathbf{D}$-EVM)}

This section is a summary of results originally presented in $[9,10]$. For the sake of brevity, only the required concepts for the purpose of this work are presented and some propositions are only enunciated. More details and formal proofs can be found in the two abovementioned references. Furthermore, in the following Sections we will make brief mentions of more concepts, applications, and algorithms, under the context of the $n \mathrm{D}-\mathrm{EVM}$, and the references where they are presented and described with enough detail.

\subsection{Preliminary Background: the n-Dimensional Orthogonal Pseudopolytopes ( $n D-O P P s$ )}

Definition 1. A singular $n$-Dimensional hyperbox in the $n$ Dimensional Euclidean Space is the continuous function

$$
\begin{aligned}
I^{n}:[0,1]^{n} & \longrightarrow[0,1]^{n} \\
x & \longmapsto I^{n}(x)=x .
\end{aligned}
$$

Definition 2. For all $i=1,2, \ldots, n$ the two singular $(n-$ 1)D hyperboxes $I_{(i, 0)}^{n}$ and $I_{(i, 1)}^{n}$ are defined as follows. If $x \in$ $[0,1]^{n-1}$ then

$$
\begin{aligned}
I_{(i, 0)}^{n}(x) & =I^{n}\left(x_{1}, \ldots, x_{i-1}, 0, x_{i}, \ldots, x_{n-1}\right) \\
& =\left(x_{1}, \ldots, x_{i-1}, 0, x_{i}, \ldots, x_{n-1}\right), \\
I_{(i, 1)}^{n}(x) & =I^{n}\left(x_{1}, \ldots, x_{i-1}, 1, x_{i}, \ldots, x_{n-1}\right) \\
& =\left(x_{1}, \ldots, x_{i-1}, 1, x_{i}, \ldots, x_{n-1}\right) .
\end{aligned}
$$

Definition 3. In a general singular $n \mathrm{D}$ hyperbox $c$ the $(i, \alpha)$ cell is defined as $c_{(i, \alpha)}=c \circ I_{(i, \alpha)}^{n}$.

Definition 4. The orientation of a cell $c \circ I_{(i, \alpha)}^{n}$ is given by $(-1)^{\alpha+i}$.

Definition 5. An $(n-1) \mathrm{D}$ oriented cell is given by the scalarfunction product $(-1)^{i+\alpha} \cdot c \circ I_{(i, \alpha)}^{n}$.

Definition 6. A formal linear combination of singular general $k \mathrm{D}$ hyperboxes, $1 \leq k \leq n$, for a closed set $A$ is called a $k$ chain. 
Definition 7 (see [11]). Given a singular $n \mathrm{D}$ hyperbox $I^{n}$ the $(n-1)$-chain, called the boundary of $I^{n}$, is given by

$$
\partial\left(I^{n}\right)=\sum_{i=1}^{n}\left(\sum_{\alpha=0,1}(-1)^{i+\alpha} \cdot I_{(i, \alpha)}^{n}\right) .
$$

Definition 8 (see [11]). Given a singular general $n \mathrm{D}$ hyperbox $c$ the $(n-1)$-chain, called boundary of $c$, is defined by

$$
\partial(c)=\sum_{i=1}^{n}\left(\sum_{\alpha=0,1}(-1)^{i+\alpha} \cdot c \circ I_{(i, \alpha)}^{n}\right) .
$$

Definition 9 (see [11]). The boundary of an $n$-chain $\sum c_{i}$, where each $c_{i}$ is a singular general $n \mathrm{D}$ hyperbox, is given by

$$
\partial\left(\sum c_{i}\right)=\sum \partial\left(c_{i}\right)
$$

Definition 10. A collection $c_{1}, c_{2}, \ldots, c_{k}, 1 \leq k \leq 2^{n}$, of general singular $n \mathrm{D}$ hyperboxes is a combination of $n \mathrm{D}$ hyperboxes if and only if

$$
\begin{aligned}
& {\left[\bigcap_{\alpha=1}^{k} c_{\alpha}\left([0,1]^{n}\right)=(\underbrace{0, \ldots, 0}_{n})\right]} \\
& \wedge\left[(\forall i, j, i \neq j, \quad 1 \leq i, \quad j \leq k)\left(c_{i}\left([0,1]^{n}\right) \neq c_{j}\left([0,1]^{n}\right)\right)\right] .
\end{aligned}
$$

In the above definition the first part of the conjunction establishes that the intersection between all the $n \mathrm{D}$ general singular hyperboxes is the origin, while the second part establishes that there are not overlapping $n \mathrm{D}$ hyperboxes.

Definition 11. An n-Dimensional Orthogonal Pseudopolytope $p$, or just an $n \mathrm{D}$-OPP $p$, will be an $n$-chain composed by $n \mathrm{D}$ hyperboxes arranged in such way that by selecting a vertex, in any of these hyperboxes, we have that such vertex describes a combination of $n \mathrm{D}$ hyperboxes composed up to $2^{n}$ hyperboxes.

\subsection{The nD-EVM: Foundations}

Definition 12 (see [10]). Let $c$ be a combination of hyperboxes in the $n$-Dimensional Euclidean Space. An Odd Edge will be an edge with an odd number of incident hyperboxes of $c$.

Definition 13 (see [10]). A brink, or extended edge, is the maximal uninterrupted segment, built out of a sequence of collinear and contiguous odd edges of an $n \mathrm{D}$-OPP.

Definition 14 (see [10]). The Extreme Vertices of an $n \mathrm{D}-\mathrm{OPP}$ $p$ are the ending vertices of all the brinks in $p$.

Definition 15 (see [10]). Let $p$ be an $n \mathrm{D}$-OPP. The Extreme Vertices Model of $p$, denoted by $\operatorname{EVM}_{n}(p)$, is defined as the model as only stores to all the extreme vertices of $p$.

\subsection{Sections and Slices of $n D-O P P s$}

Definition 16. Let $p$ be an $n \mathrm{D}$-OPP. A $k \mathrm{D}$ couplet of $p, 1<k<$ $n$, is the maximal set of $k \mathrm{D}$ cells of $p$ that lies in a $k \mathrm{D}$ space, such that a $k \mathrm{D}$ cell $e_{0}$ belongs to a $k \mathrm{D}$ extended hypervolume if and only if $e_{0}$ belongs to an $(n-1) \mathrm{D}$ cell present in $\partial(p)$.

Definition 17. The Projection Operator for $(n-1) \mathrm{D}$ cells, points, and set of points is, respectively, defined as follows.

(i) Let $c\left(I_{(i, \alpha)}^{n}(x)\right)=\left(x_{1}, \ldots, x_{n}\right)$ be an $(n-1) \mathrm{D}$ cell embedded in the $n \mathrm{D}$ space. $\pi_{j}\left(c\left(I_{(i, \alpha)}^{n}(x)\right)\right)$ will denote the projection of the cell $c\left(I_{(i, \alpha)}^{n}(x)\right)$ onto an $(n-1) \mathrm{D}$ space embedded in $n \mathrm{D}$ space whose supporting hyperplane is perpendicular to $X_{j}$-axis: $\pi_{j}\left(c\left(I_{(i, \alpha)}^{n}(x)\right)\right)=\left(x_{1}, \ldots, \widehat{x}_{j}, \ldots, x_{n}\right)$.

(ii) Let $v=\left(x_{1}, \ldots, x_{n}\right)$ a point in the $n \mathrm{D}$ space. The projection of $v$ in the $(n-1) \mathrm{D}$ space, denoted by $\pi_{j}(v)$, is given by $\pi_{j}(v)=\left(x_{1}, \ldots, \widehat{x}_{j}, \ldots, x_{n}\right)$.

(iii) Let $Q$ be a set of points in the $n \mathrm{D}$ space. The projection of the points in $Q$, denoted by $\pi_{j}(Q)$, is defined as the set of points in the $(n-1) \mathrm{D}$ space such that $\pi_{j}(Q)=$ $\left\{p \in \mathfrak{R}^{n-1}: p=\pi_{j}(x), x \in Q \subset \mathfrak{R}^{n}\right\}$.

Where $\widehat{x}_{j}$ is the coordinate corresponding to $X_{j}$-axis to be suppressed.

\section{Definition 18. Consider an $n \mathrm{D}-\mathrm{OPP} p$.}

(i) Let $n p_{i}$ be the number of distinct coordinates present in the vertices of $p$ along $X_{i}$-axis, $1 \leq i \leq n$.

(ii) Let $\Phi_{k}^{i}(p)$, or just $\Phi_{k}^{i}$ if no reference to $p$ is needed, be the $k$ th $(n-1) \mathrm{D}$ couplet of $p$ which is perpendicular to $X_{i}$-axis, $1 \leq k \leq n p_{i}$.

Definition 19. A Section is the $(n-1) \mathrm{D}-\mathrm{OPP}, n>1$, resulting from the intersection between an $n \mathrm{D}-\mathrm{OPP} p$ and a $(n-1) \mathrm{D}$ hyperplane perpendicular to the coordinate $X_{i}$-axis, $1 \leq i \leq$ $n$, which not coincide with any $(n-1) \mathrm{D}$-couplet of $p$. A section will be called external or internal section of $p$ if it is empty or not, respectively. $S_{k}^{i}(p)$ will refer to the $k$ th section of $p$ between $\Phi_{k}^{i}(p)$ and $\Phi_{k+1}^{i}(p), 1 \leq k<n p_{i}$.

Theorem 20 (see [10]). The projection of the set of $(n-1) D$ couplets, $\pi_{i}\left(\Phi_{k}^{i}(p)\right)$, of an $n D-O P P p$, can be obtained by computing the regularized $X O R(\otimes)$ between the projections of its previous $\pi_{i}\left(S_{k-1}^{i}(p)\right)$ and next $\pi_{i}\left(S_{k}^{i}(p)\right)$ sections; that is,

$$
\pi_{i}\left(\Phi_{k}^{i}(p)\right)=\pi_{i}\left(S_{k-1}^{i}(p)\right) \otimes^{*} \pi_{i}\left(S_{k}^{i}(p)\right), \quad \forall k \in\left[1, n p_{i}\right] .
$$

Theorem 21 (see [10]). The projection of any section, $\pi_{i}\left(S_{k}^{i}(p)\right)$, of an $n D-O P P p$, can be obtained by computing the regularized XOR between the projection of its previous section, $\pi_{i}\left(S_{k-1}^{i}(p)\right)$, and the projection of its previous couplet $\pi_{i}\left(\Phi_{k}^{i}(p)\right)$.

\subsection{The Regularized XOR Operation on the $n D-E V M$}

Theorem 22 (see [9]). Let $p$ and $q$ be two nD-OPPs having $\operatorname{EVM}_{n}(p)$ and $\operatorname{EVM}_{n}(q)$ as their respective EVMs in $n D$ space; then

$$
\operatorname{EVM}_{n}\left(p \otimes^{*} q\right)=\operatorname{EVM}_{n}(p) \otimes \operatorname{EVM}_{n}(q) .
$$




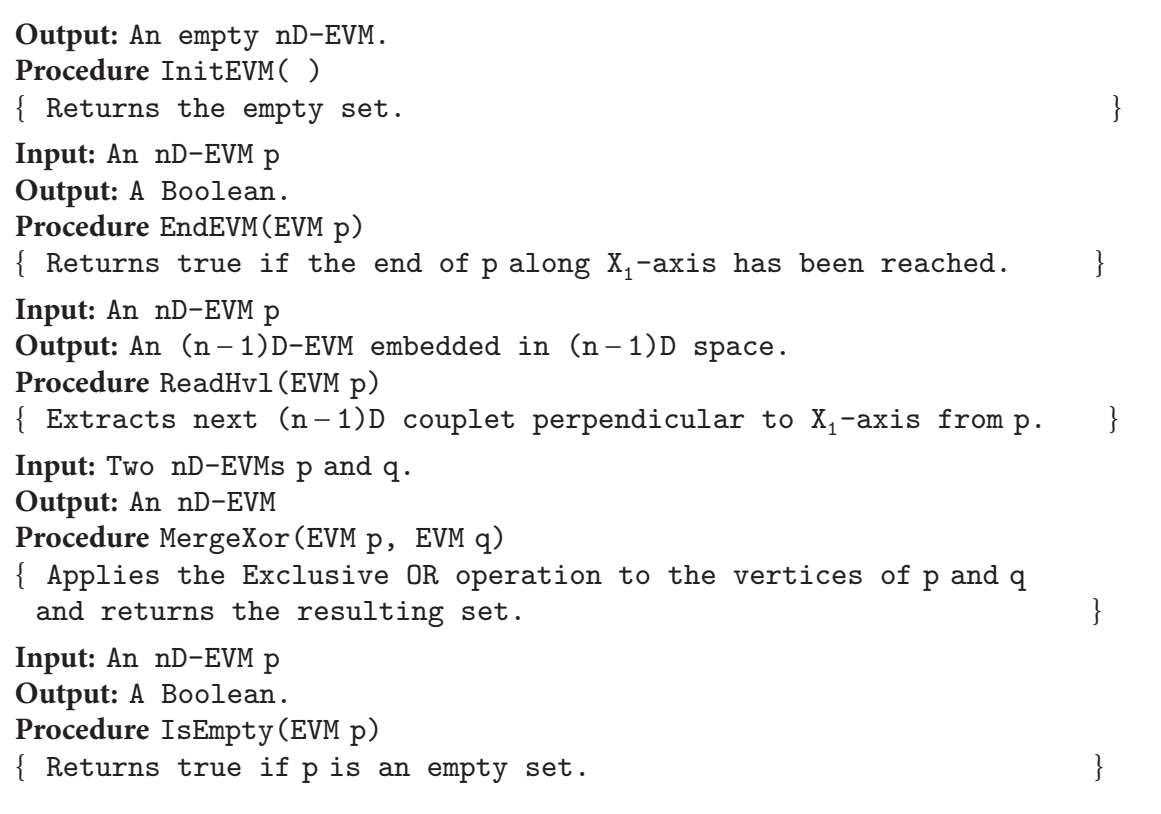

Algorithm 1: Some primitive operations in the $n \mathrm{D}$-EVM.

This result allows expressing a formula for computing $n \mathrm{D}$ OPPs' sections from couplets and vice-versa, by means of their corresponding Extreme Vertices Models. These formulae are obtained by combining Theorem 22 with Theorem 20, and Theorem 22 with Theorem 21, respectively.

Corollary 23 (see [9]).

$$
\begin{aligned}
& \operatorname{EVM}_{n-1}\left(\pi_{i}\left(\Phi_{k}^{i}(p)\right)\right) \\
& \quad=E V M_{n-1}\left(\pi_{i}\left(S_{k-1}^{i}(p)\right)\right) \otimes E V M_{n-1}\left(\pi_{i}\left(S_{k}^{i}(p)\right)\right) .
\end{aligned}
$$

Corollary 24 (see [9]).

$$
\begin{aligned}
& \operatorname{EVM}_{n-1}\left(\pi_{i}\left(S_{k}^{i}(p)\right)\right) \\
& \quad=\operatorname{EVM}_{n-1}\left(\pi_{i}\left(S_{k-1}^{i}(p)\right)\right) \otimes E V M_{n-1}\left(\pi_{i}\left(\Phi_{k}^{i}(p)\right)\right) .
\end{aligned}
$$

Finally, the following corollary can be stated, which correspond to a specific situation of the XOR operand. It allows computing the union of two $n \mathrm{D}$-OPPs when that specific situation is met.

Corollary 25 (see [9]). Let $p$ and $q$ be two disjoint or quasidisjoint $n D$-OPPs having $\operatorname{EVM}_{n}(p)$ and $\operatorname{EVM}_{n}(q)$ as their respective Extreme Vertices Models; then

$$
\operatorname{EVM}_{n}(p \cup q)=\operatorname{EVM}_{n}(p) \otimes \operatorname{EVM}_{n}(q)
$$

2.5. Basic Algorithms for the nD-EVM. According to previous sections the primitive operations shown in Algorithm 1 can be defined based in the functions originally presented in [9].
Function MergeXor, according to Theorem 22, performs an $\mathrm{XOR}$ between two $n \mathrm{D}$-EVMs; that is, it keeps all vertices belonging to either $\operatorname{EVM}_{n}(p)$ or $\operatorname{EVM}_{n}(q)$ and discards any vertex that belongs to both $\operatorname{EVM}_{n}(p)$ and $\operatorname{EVM}_{n}(q)$. Since the model is sorted, this function consists of a simple merginglike algorithm, and therefore, it runs on linear time $[9,10]$.

From the above primitive operations, Algorithms 2 and 3 are derived. Algorithm 4 computes the sequence of sections of an $n \mathrm{D}$-OPP $p$ from its $n \mathrm{D}$-EVM using the previous functions. It sequentially reads the projections of the $(n-1) \mathrm{D}$ couplets $h v l$ of polytope $p$. Then it computes the sequence of sections using function GetSection. Each pair of sections $S_{i}$ and $S_{j}$ (the previous and next sections about the current $h v l$ ) is processed by a generic processing procedure (called Process), which performs the desired actions upon $S_{i}$ and $S_{j}$.

\section{Representing Hypervoxelization Datasets through $n$ D Orthogonal Polytopes}

The conversion of a $(n-1) \mathrm{D}$ hypervoxelization to a $n \mathrm{D}$ polytope, and therefore to a $n \mathrm{D}-\mathrm{EVM}$, is in fact a straight procedure. The methodology to be described in this Section was originally presented, for the $4 \mathrm{D}$ case, in [12]. As commented in Section 1, the proposal is oriented to color spaces with three or more values. If it is the case that datasets have binary intensity values, then refer to [4], where a more appropriate representation is described. It is assumed the intensities of each hypervoxel are expressed as a single number.

A $n \mathrm{D}$ hyperprism is a polytope generated by the parallel motion of an $(n-1) \mathrm{D}$ polytope; it is bounded by the $(n-1) \mathrm{D}$ polytope in its initial and final positions and by several $(n-$ $1) \mathrm{D}$ hyperprisms $[13,14]$. In this sense, each hypervoxel in a $(n-1) \mathrm{D}$ dataset is extruded towards the $n$th Dimension; 
Input: An ( $n-1) D-E V M$ corresponding to section $S$.

An $(n-1) D-E V M$ corresponding to couplet hvl.

Output: An $(n-1) D-E V M$.

Procedure GetSection(EVM S, EVM hvl)

end-of-procedure

$1 /$ Returns the projection of the next section of an $\mathrm{nD}-\mathrm{OPP}$ whose previous section is $\mathrm{S}$. return MergeXor ( $\mathrm{S}, \mathrm{hvl}$ )

Algorithm 2: Computing sections from couplets.

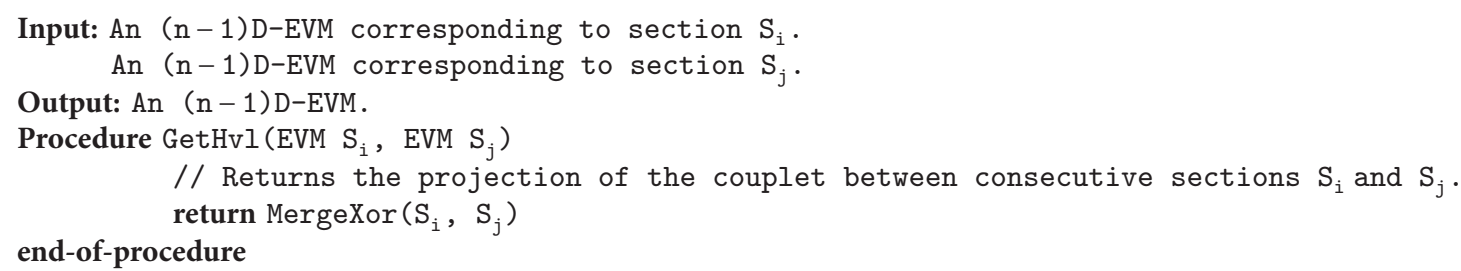

Algorithm 3: Computing couplets from sections.

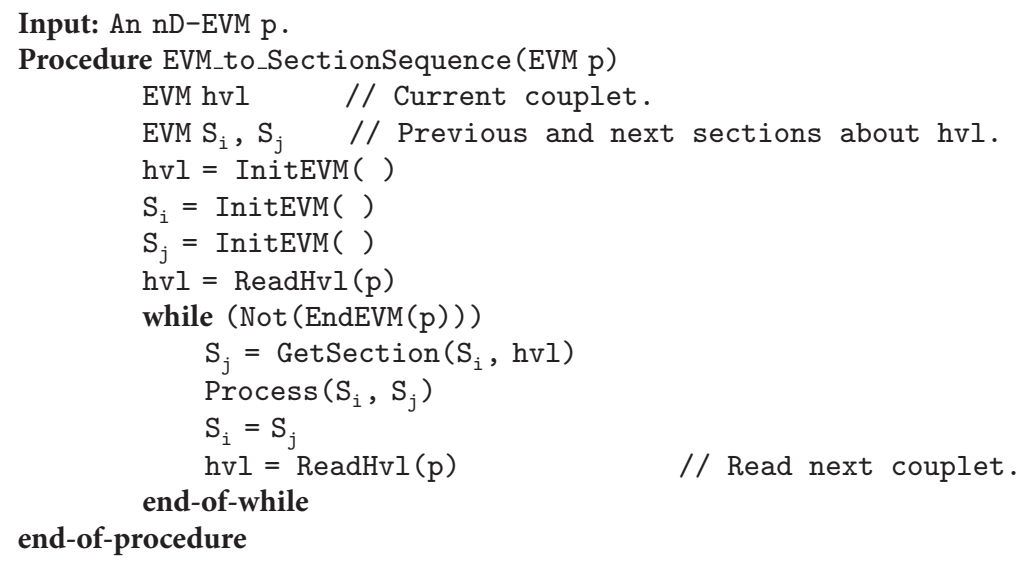

Algorithm 4: Computing the sequence of sections from an $n$ D-OPP $p$ represented through the $n$ D-EVM.

that is, it is converted in a $n \mathrm{D}$ hyperprism whose bases are precisely the original hypervoxel and its height is given by its corresponding intensity-plus-one value in the dataset. The vertices' coordinates $X_{1}, X_{2}, \ldots, X_{n-1}$ in the hyperprism's bases correspond to the original hypervoxel's coordinates. The inferior base's points have their $X_{n}$ coordinate equal to zero, while in the remaining vertices the $X_{n}$ coordinate is equal to the intensity-plus-one value (see Figure 1 for an example under the instance $n=4)$. Let us call $x f$ to the set composed by the $n \mathrm{D}$ hyperprisms (the extruded hypervoxels) of the extruded $(n-1) \mathrm{D}$ dataset.

Let $\mathrm{pr}_{i}$ be a $n \mathrm{D}$ hyperprism in $x f$ and $n \mathrm{pr}$ the number of prisms in that set: $n \mathrm{pr}$ is in fact equal to the number of hypervoxels in the original dataset. Since all hyperprisms in $x f$ are quasidisjoint $n \mathrm{D}$-OPPs, the extreme vertices, of the whole $n \mathrm{D}$ extruded dataset, can be easily obtained by computing the regularized union of all the hyperprisms in $x f$. Hence, Corollary 25 is applied in the following way:

$$
\operatorname{EVM}_{n}(F)=\bigotimes_{i=1}^{n \mathrm{pr}} \operatorname{EVM}_{n}\left(\mathrm{pr}_{i} \in x f\right)
$$

where $F$ is the $n \mathrm{D}$-OPP that represents the union of all the hyperprisms in $x f$. By this way, it is obtained a representation for a $(n-1) \mathrm{D}$ Dataset through a $n \mathrm{D}$-OPP and the $n \mathrm{D}$-EVM.

Now we describe some first results related to the conversion from voxelizations (3D hypervoxelizations) to our proposed representation. Such datasets were taken from The Volume Library [15], and the University of Iowa's Department of Radiology [16]. Datasets' visualizations shown in Table 1 were obtained via a volume rendering software available at [17]. Table 1 also shows the space complexity measures 


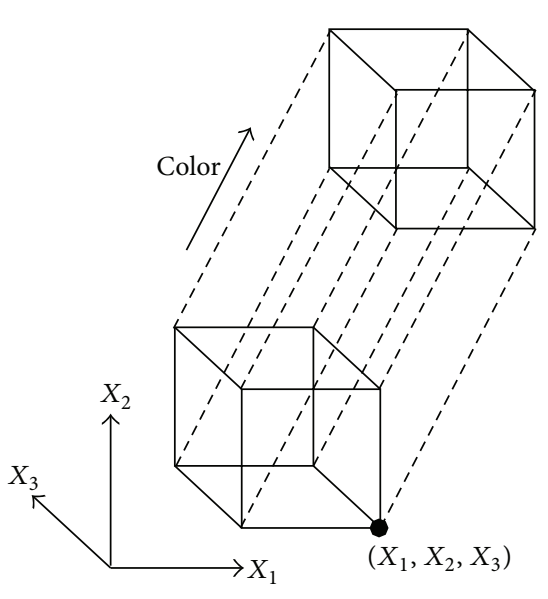

(a)

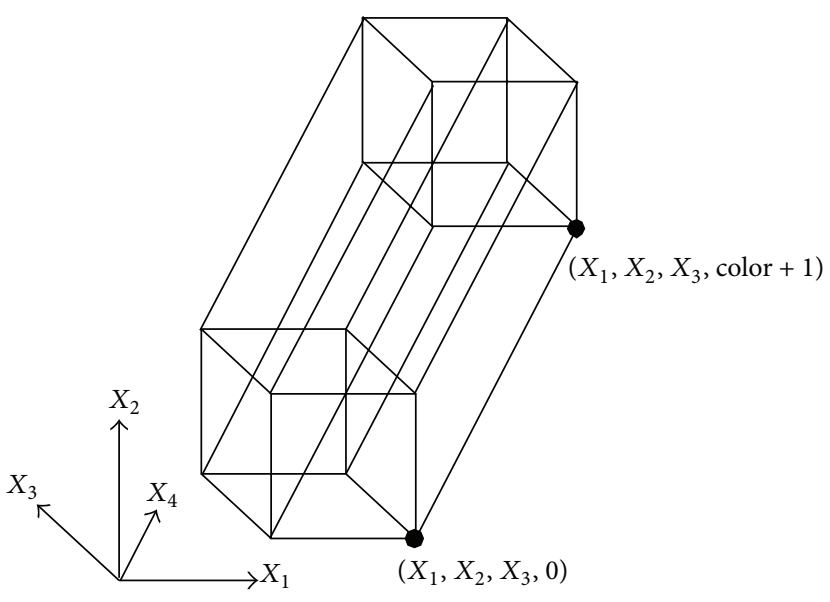

(b)

FIGURE 1: Extruding a voxel towards the fourth dimension. The result is a $4 \mathrm{D}$ hyperprism whose height is determined by the voxel's intensity color.

TABLE 1: Volume datasets used for conversion to 4D-OPPs and to be finally expressed through the 4D-EVM.

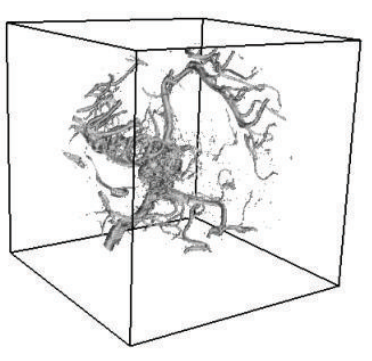

Aneurism

Description: rotational C-arm X-ray scan of the arteries of the right half of a human head. A contrast agent was injected into the blood and an aneurism is present.

Voxelization size:

$(256 \times 256 \times 256) \equiv 16,777,216$

4D-EVM Size: $1,317,008$

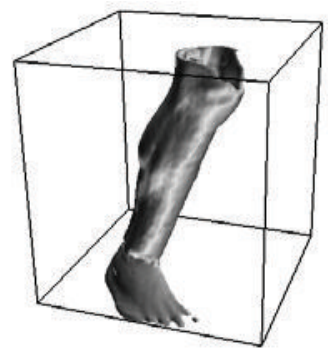

Statue Leg

Description: CT scan of a leg of a bronze statue.

Voxelization size:
$(341 \times 341 \times 93) \equiv 10,814,133$
4 D-EVM Size: $8,858,034$

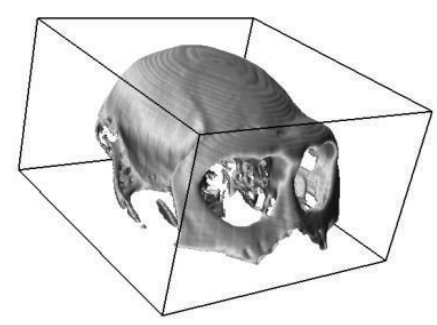

CTMonkey

Description: registered Monkey Head

Voxelization size:
$(256 \times 256 \times 62) \equiv 4,063,232$
4D-EVM Size: $3,573,172$ obtained when the considered voxelizations were converted to $4 \mathrm{D}$-OPPs and the $n \mathrm{D}$-EVM. Now, let $p$ be a dataset expressed under a $(n-1) \mathrm{D}$ hypervoxelization with size $\left(x_{1}\right.$ Size $\times x_{2}$ Size $\times \cdots \times x_{n-1}$ Size $)$ and with $\operatorname{EVM}_{n}(p)$ as its corresponding $n \mathrm{D}-\mathrm{EVM}$. Consider the ratio [12]:

$$
\frac{x_{1} \text { Size } \cdot x_{2} \text { Size } \cdots x_{n-1} \text { Size }}{\operatorname{Card}\left(\operatorname{EVM}_{n}(p)\right)}
$$

For example, see model Aneurism (Table 1). Its source voxelization has size $(256 \times 256 \times 256)$ which implies that it is required to store $16,777,216$ voxels. The $4 \mathrm{D}$-EVM associated to Aneurism has 1,317,008 extreme vertices. Hence, the proposed ratio gives the value 12.7388 which tell us the number of stored voxels that belong to the original representation of the object is precisely 12.7388 times greater than the number of obtained extreme vertices. Table 2 shows the ratio Number-of-voxels/Number-of-Extreme-Vertices for the models described in Table 1 . The value shared by the ratio depends on the topology and geometry of the objects being modeled, but it shows how some conciseness is gained, related to storing requirements, when they are represented through the $n \mathrm{D}$-EVM.

Tables 3, 4, and 5 show some 3D couplets obtained from datasets Aneurism, CT-Monkey, and Statue Leg, respectively. 3D-EVM visualizations from Tables 3 to 6 were achieved by means of visualization software developed in [18-20]. Table 6 shows some $2 \mathrm{D}$ sections corresponding to one of the $3 \mathrm{D}$ couplets from the model CT-Monkey. There are shown, by direct application of Algorithm 4, 22 of these 2D sections which allow understand the internal organization of the selected 3D couplet. It is possible to visualize some internal bone structures associated to the monkey's cranium.

In [21] is presented an $n \mathrm{D}$-EVM-based algorithm for computing Connected Components Labeling (CCL) over an $n \mathrm{D}$-OPP. It is well known CCL is one of the most important 
TABLE 2: The ratio number of voxels/number of extreme vertices for datasets shown in Table 1.

\begin{tabular}{lccc}
\hline Object $p$ & $\begin{array}{c}\text { Voxelization size } \\
\text { (number of voxels) }\end{array}$ & $\begin{array}{c}\text { Card }\left(\mathrm{EVM}_{4}(p)\right) \\
\text { (number of extreme vertices) }\end{array}$ & $\frac{x_{1} \text { Size } \cdot x_{2} \text { Size } \cdot x_{3} \text { Size }}{\text { Card }\left(\mathrm{EVM}_{4}(p)\right)}$ \\
\hline Aneurism & $16,777,216$ & $1,317,008$ & 12.7388 \\
Statue Leg & $10,814,133$ & $8,858,034$ & 1.2208 \\
CT Monkey & $4,063,232$ & $3,573,172$ & 1.1371 \\
\hline
\end{tabular}

TABLE 3: Some 3D Couplets, perpendicular to color axis, extracted from the 4D-EVM associated to dataset Aneurism.

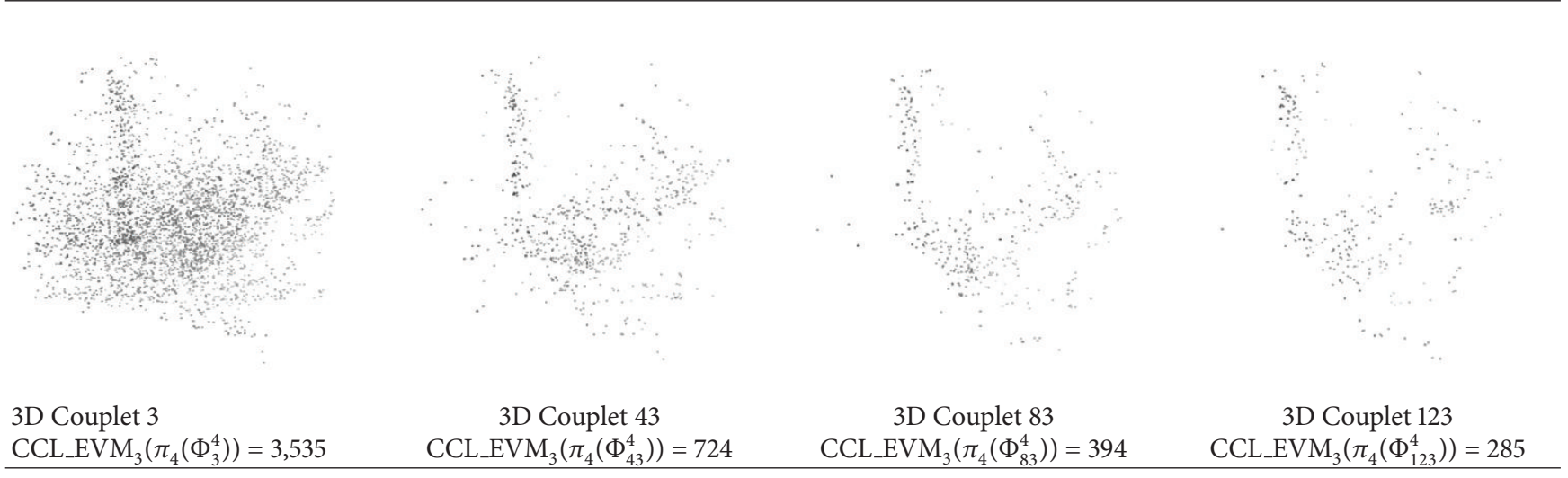

CCL_EVM ${ }_{3}\left(\pi_{4}\left(\Phi_{3}^{4}\right)\right)=3,535$ CCL_EVM ${ }_{3}\left(\pi_{4}\left(\Phi_{43}^{4}\right)\right)=724$ CCL_EVM ${ }_{3}\left(\pi_{4}\left(\Phi_{83}^{4}\right)\right)=394$

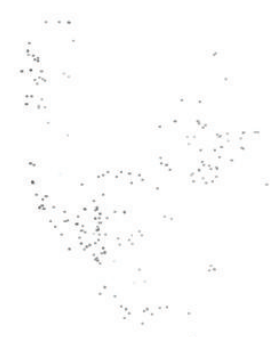

3D Couplet 163

3D Couplet 203

$\mathrm{CCL}_{-} \mathrm{EVM}_{3}\left(\pi_{4}\left(\Phi_{163}^{4}\right)\right)=210$

$\mathrm{CCL}_{-} \mathrm{EVM}_{3}\left(\pi_{4}\left(\Phi_{203}^{4}\right)\right)=192$

tools in image processing and computer vision. The main idea is to assign labels to the pixels in an image such that all pixels that belong to a same Connected Component (CC) have the same label $[22,23]$. The direct application of our CCL-EVM-Based algorithm over the 4D-EVMs associated to our three datasets will report only one component. This is because of the way the original $4 \mathrm{D}$-OPPs were built (see (13)). Now, let CCL_EVM $\mathrm{EV}_{3}(p)$ be the output shared by our mentioned CCL-EVM-Based algorithm when is sent as input a $3 \mathrm{D}$-OPP $p$, expressed under the $n \mathrm{D}$-EVM. In other words, CCL_EVM $\mathrm{EM}_{3}(p)$ gives the number of $3 \mathrm{D}$ CCs present in p. For specific details about our CCL-EVM-Based algorithm refer to [21]. In Tables 3 to 5 we also make mention of the number of CCs present in the shown $3 \mathrm{D}$ couplets. These data is going to be part of an analysis to be performed in Section 6 .

The importance behind a dataset is the information can be obtained from it. If the datasets are represented through the $n \mathrm{D}$-EVM then the extraction of its couplets perpendicular to $X_{n}$-axis will provide a classification of the elements in the original model according to their color intensities. Or in others words, we obtain a dataset's segmentation based exclusively on color intensities. Given a dataset based on a scale of $K$ intensities, then it is possible to obtain at most, from its corresponding $n \mathrm{D}-\mathrm{OPP}, K+1(n-1) \mathrm{D}$ couplets perpendicular to the axis associated to color. The "extra" couplet, in fact the first couplet, is the result of the union of all the inferior bases of the $n \mathrm{D}$ hyperprisms in $x f$ (the set composed by the dataset's extruded hypervoxels): the points in such bases have value zero for the coordinate associated to color. Under this reasoning, Tables 3, 4, and 5 present part of the color-intensities-based segmentation achieved in our considered datasets.

It is possible that color intensities correspond to physical properties. For example, in a 3D medical dataset intensities could refer to certain tissues. Hence, by extracting $3 \mathrm{D}$ couplets perpendicular to color axis we could obtain, for a given couplet, only those parts of the $3 \mathrm{D}$ dataset with the same type of tissue. The type of material is, in this case, assumed to be defined by the color intensity of the voxels in the original dataset. The projection of each couplet, perpendicular to the axis associated to color, in a $n \mathrm{D}-\mathrm{EVM}$, is in fact a $(n-1) \mathrm{D}-\mathrm{EVM}$ which in time corresponds to a binary 
TABLE 4: Some 3D Couplets, perpendicular to color axis, extracted from the 4D-EVM associated to dataset CT-Monkey.

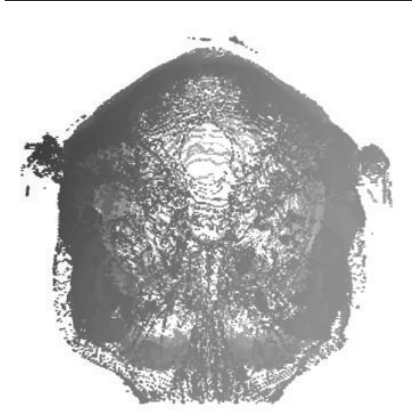

3D Couplet 3

CCL_EVM $3\left(\pi_{4}\left(\Phi_{3}^{4}\right)\right)=22,892$

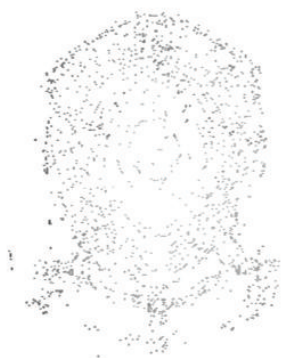

3D Couplet 143

$\mathrm{CCL}_{-} \mathrm{EVM}_{3}\left(\pi_{4}\left(\Phi_{143}^{4}\right)\right)=1,548$

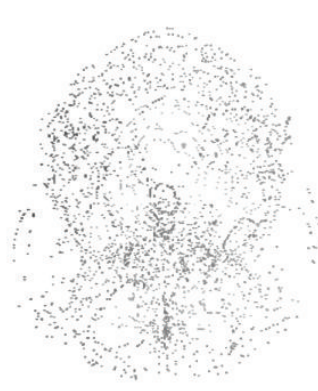

3D Couplet 23

CCL_EVM ${ }_{3}\left(\pi_{4}\left(\Phi_{23}^{4}\right)\right)=2,623$

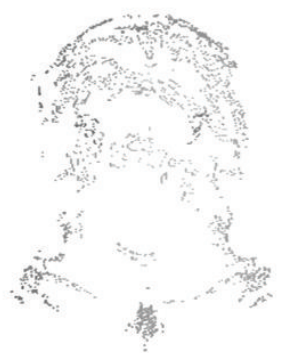

3D Couplet 173

CCL_EVM ${ }_{3}\left(\pi_{4}\left(\Phi_{173}^{4}\right)\right)=1,558$

TABLE 5: Some 3D Couplets, perpendicular to color axis, extracted from the 4D-EVM associated to dataset Statue Leg.

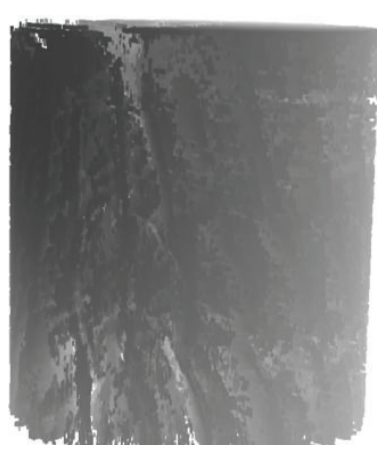

3D Couplet 3

CCL_EVM ${ }_{3}\left(\pi_{4}\left(\Phi_{3}^{4}\right)\right)=113,237$

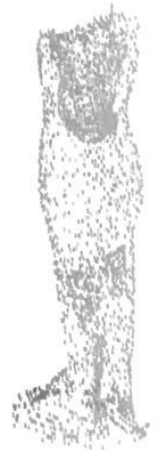

3D Couplet 23

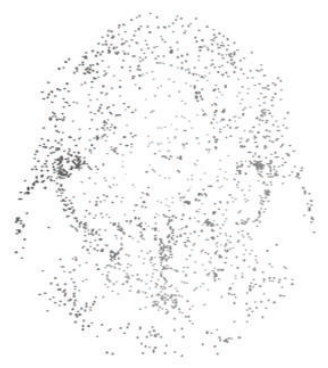

3D Couplet 63

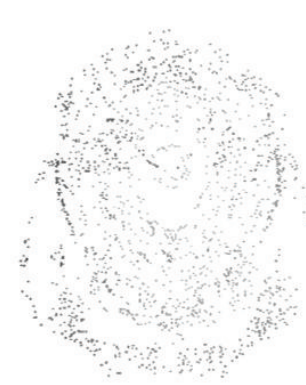

3D Couplet 103

.


TABLE 6: Visualizing some 2D Sections perpendicular to $X_{1}$-axis which were processed from a 3D Couplet of the $4 \mathrm{D}$-OPP associated to dataset CT-Monkey.

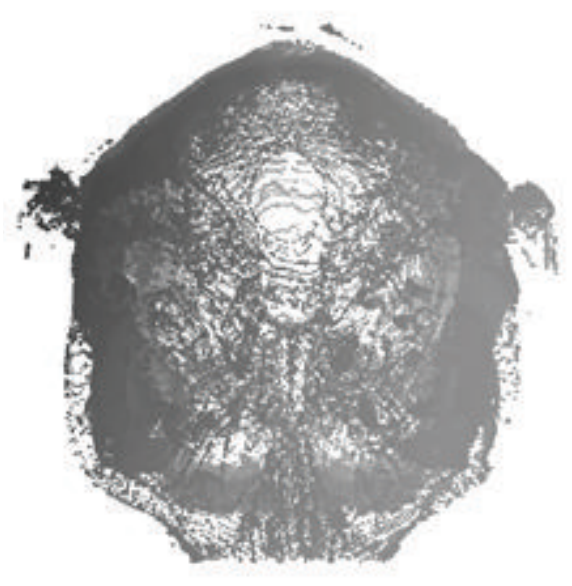

3D Couplet 18

2D Section 1


dataset because it only contains material of the same type. Hence, it is possible to apply Algorithm 4 to these $(n-1) \mathrm{D}$ couplets in order to obtain their associated $(n-2) \mathrm{D}$ sections. These sections will share a description of the interior of the homogeneous object with the objective of performing the appropriate analyses according to the application, as seen previously in the example described in Table 6.

This Section has focused about the representation of hypervoxelizations through $n \mathrm{D}$-OPPs which in time are expressed in the $n \mathrm{D}$-EVM. Moreover, we have seen how the basic procedures under the Extreme Vertices Model share the extraction of useful information related to the original datasets: the classification of their elements according to intensities via $(n-1) \mathrm{D}$ couplets perpendicular to color axis. Likewise, it is possible to compute other geometrical and topological interrogations over an $n \mathrm{D}$-EVM. By this way it could be obtained much more information and properties about the datasets. Furthermore, there are well specified procedures under the $n \mathrm{D}-\mathrm{EVM}$ which allow performing Regularized Boolean Operations, Polytopes Splitting, Discrete Compactness Computation, Morphological Operations, Connected Components Labeling, Boundary Extraction, among others. In [9, 10, 12, 21, 24, 25] there are described with enough detail algorithms based in the $n \mathrm{D}$ EVM which are useful and efficient for performing these interrogations and/or manipulations.

According to the above discussion, if datasets are represented through the $n \mathrm{D}-\mathrm{EVM}$ then the extraction of its couplets will provide a classification of the elements in the original model according to their color intensities. The projection of each couplet, perpendicular to the axis associated to color is in fact a $(n-1) \mathrm{D}$-EVM which in time corresponds to a binary dataset because it only contains material of the same type, in this case, the same intensity. We are now in a critical point where the next logical step is given by the consideration of an additional approach in such way that the points in a dataset could be characterized by taking in account not only their color intensity but their class. Due to the presence of scanning noise and artifacts, a classification based only in intensities is sometimes not enough because points that should belong to the same class could be characterized as distinct points and therefore assigned to distinct classes, hence, our $n \mathrm{D}$ representation will integrate each point in distinct $(n-$ 1)D couplets. Let an intelligent approach, such as an Artificial Neural Network, be responsible for automatically identifying the classes of points present in a multidimensional dataset by taking in account their geometry, topology, neighborhood, and so forth. Once the classification is achieved, then the corresponding conversion to our proposed $n$-Dimensional representation could take place. It should be possible to assign to each class a coordinate on the $n$th dimension in such way $(n-1) \mathrm{D}$ couplets perpendicular to the $n$th axis now correspond to sets of points, in the original dataset, that belong to the same class. It is also hypothesized that by considering more appropriate points classification, a much better conciseness should be obtained. This is the line or reasoning which is going to be explored in the following Sections.

\section{A Survey on 1-Dimensional Kohonen Networks (1D-KNs)}

A Kohonen Network with $\psi$ inputs and $O$ neurons may be used to classify points embedded in an $\psi$-Dimensional space into $O$ categories $[26,27]$. Input points have the form $\left(x_{1}, \ldots, x_{i}, \ldots, x_{\psi}\right)$. Each neuron $j, j=1,2, \ldots, O$, has associated an $\psi$-Dimensional Weights Vector which has a representation of its corresponding class $\kappa_{j}$. All these vectors have the form $W_{j}=\left(w_{j, 1}, \ldots, w_{j, \psi}\right), j=1,2, \ldots, O$.

A set of training points are presented to the network $T$ times. According to [28], all values of Weight Vectors should be randomly initialized. In the $t$ th presentation, $t=1,2$, $\ldots, T$, the neuron whose Weights Vector $W_{j}, 1 \leq j \leq O$, is the most similar to the input point $P$ is chosen as Winner Neuron. In the model proposed by Kohonen, such selection is based on the Squared Euclidean Distance. The selected neuron is that with the minimal distance between its Weights Vector and the input point $P$ :

$$
d_{j}=\sum_{i=1}^{\psi}\left(P_{i}-W_{j, i}(t)\right)^{2}, \quad 1 \leq j \leq O .
$$

Once the $\ell$ th Winner Neuron, $1 \leq \ell \leq O$, in the $t$ th presentation, has been identified each one of the network's weights vectors is updated according to:

$$
\begin{array}{r}
W_{j, i}(t+1)=W_{j, i}(t)+\frac{1}{t+1} \varphi(j, \ell)\left[P_{i}-W_{j, i}(t)\right], \\
i=1,2, \ldots, \psi, \quad j=1,2, \ldots, O,
\end{array}
$$

where the term $1 /(t+1)$ is the learning coefficient and $\varphi(j, \ell)$ is a neighborhood function that denotes the distance between the Winner Neuron $\ell$ and the neuron $j$. For neurons close enough to the winner neuron, $\varphi(j, \ell)$ should be a value near to 1. On the other hand, $\varphi(j, \ell)$ is close to zero for those neurons characterized as distant to the Winner Neuron.

When the $T$ presentations have been achieved, the values of the Weights Vectors correspond to coordinates of the "gravity centers" of the clusters of the $O$ categories.

\section{Multidimensional Hypervoxelizations' Segmentation Achieved by 1D-KNs}

Nonsupervised segmentation of datasets has great potential in the practice, but this task is complex for several reasons, being one of them, for example, the datasets' acquisition process which could introduce noise and artifacts which are difficult to correct. As commented previously, the specific problem now to be under attack is the automatic nonsupervised segmentation based on the assistance of a $1 \mathrm{D}-\mathrm{KN}$. It is expected there could be identified, during its training processes, the proper representations for a previously established number of classes. Hence, a dataset can be segmented in such way each type of region is appropriately identified. Many methods for description, object recognition or indexing are sustained on a preprocessing based on automatic segmentation [22, 29, 30]. This Section describes our methodology which is inspired in some facts established originally in [31]. 
TABLE 7: Additional volume datasets to be considered for automatic segmentation via a 1D Kohonen Network.

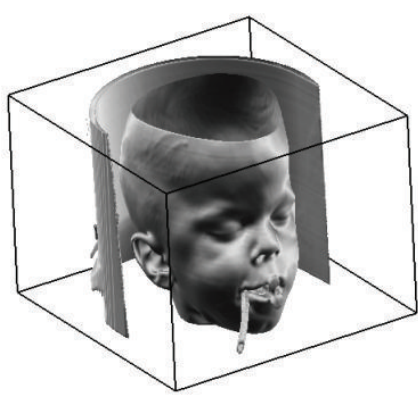

$V L-B a b y$

Description: baby head.

Voxelization size: $(256 \times 256 \times 98) \equiv 6,422,528$

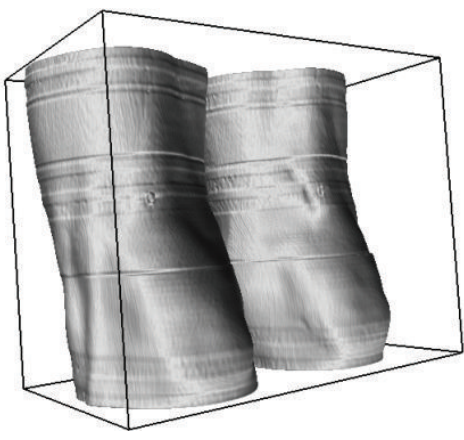

VL-CT-Knee

Description: computed tomography of knee, anterior tibial osteotomy.

Voxelization size: $(379 \times 229 \times 305) \equiv 26,471,255$
An aspect to be considered respect to the training sets to be used is the fact a first approach could suggest that the color intensity of each hypervoxel in a dataset can be seen as an input vector (formerly an input scalar). However, as discussed in several works, such as $[32,33]$, a network will be biased towards a characterization based only in intensities. It is clear each hypervoxel has an intensity which, it is understood, captures, or is associated, to a particular property (a type of tissue, material, etc.); however, it is important to consider the hypervoxels around it. The neighborhood surrounding a given hypervoxel complements the information about the properties to be identified. Let $v$ be a hypervoxel in an $(n-1) \mathrm{D}$ dataset, $n \geq 2$. Given $v$ it is possible to build a subdataset by taking those hypervoxels inside a hypercubical neighborhood of radius $r$ and center at $v$. Hypervoxel $v$ and its neighboring hypervoxels will be called a $(n-1)$-Dimensional mask. For example, given a $3 \mathrm{D}$ dataset, if we refer to $3 \mathrm{D}$ masks of radius $r=2$ then we have subdatasets of size $(5 \times 5 \times 5)$ whose central voxel, with coordinates $(r+1, r+1, r+1)$, is the one of interest and the remaining 124 voxels compose its cubical neighborhood.

Now, the network's training set is then composed by all the $(n-1) \mathrm{D}$ masks of radius $r$ that can be generated in a given $(n-1) \mathrm{D}$ dataset. As commented in Section 4 a 1D-KN expects as input a vector, or point, embedded in the $\psi$-Dimensional Space. A $(n-1) \mathrm{D}$ mask is formerly seen as a $(n-1) \mathrm{D}$ array, but it is clear by stacking its columns on top of one another a vector is obtained. This straightforward procedure linearizes a $(n-1) \mathrm{D}$ mask making it a suitable input for the network. The resulting vectors, composed by

$$
\psi=(2 r+1)^{(n-1)}
$$

components, preserve the properties associated to the original $(n-1) \mathrm{D}$ masks. Each component has the color intensity of the corresponding hypervoxel in its associated $(n-1) \mathrm{D}$ mask. By this way, we have specified the elements that conform our proposed approach for automatic segmentation of $(n-$ 1)D hypervoxelizations. We proceed now to describe some experiments and the obtained results.
Our study cases were based in the set of 3D datasets presented in Section 3 plus two additional datasets described in Table 7. Both datasets were taken from The Volume Library [15] and the visualizations shown in Table 7 were achieved via a volume rendering software available at [17].

The implemented 1D-KNs have the following topologies and training conditions:

(i) 3D masks radius: $r=2$,

(ii) inputs: $\psi=5 \times 5 \times 5=125$,

(iii) neurons (classes): $O=40$,

(iv) presentations: $T=10$.

According to (16) we must define a neighborhood function. In this work we will use the following rule:

$$
\varphi(j, \ell)= \begin{cases}1, & j=\ell, \\ 0, & j \neq \ell .\end{cases}
$$

That is, once the Winner Neuron $\ell$ has been identified, only its weights are updated as established in (16). As commented above, training sets' cardinalities clearly depend on the value for radius $r$, but also relies on the dataset's size. Because we are considering five testing voxelizations, each one to be managed by its corresponding $1 \mathrm{D}-\mathrm{KN}$, then we have to think about five different training sets. In Table 8 we can see the corresponding cardinalities of the training sets associated to each considered voxelization.

Table 9 shows the obtained distribution of the training 3D masks in each one of the available 40 classes. Firstly, according to this Table, the networks trained with 3D masks associated to datasets Aneurism, VL-CT-Knee, and VL-Baby, distributed all their $3 \mathrm{D}$ masks along their 40 classes. In the case for networks trained with 3D masks associated to datasets Statue Leg and CT Monkey only 22 and 39 classes are, respectively, used. A second observation arises: in all the proposed networks there is a class which has associated more 
TABLE 8: Training sets' cardinalities for voxelizations described in Tables 1 and 7.

\begin{tabular}{lcc}
\hline Dataset & $\begin{array}{c}\text { Voxelization size } \\
\text { (number of voxels) }\end{array}$ & $\begin{array}{c}\text { Training set's } \\
\text { cardinality }\end{array}$ \\
\hline Aneurism & $16,777,216$ & $16,003,008$ \\
Statue Leg & $10,814,133$ & $10,107,641$ \\
CT Monkey & $4,063,232$ & $3,683,232$ \\
VL-CT-Knee & $26,471,255$ & $25,396,875$ \\
VL-Baby & $6,422,528$ & $5,969,376$ \\
\hline
\end{tabular}

than $45 \%$ of the elements in its corresponding training set. Seeing Table 9 it is possible to identify such classes as follows.

(i) Dataset Aneurism: class 4, 98.47\%.

(ii) Dataset Statue Leg: class 24, 93.72\%.

(iii) Dataset CT Monkey: class 27, 81.69\%.

(iv) Dataset VL-CT-Knee: class 27, 48.17\%.

(v) Dataset VL-Baby: class 9, 67.16\%.

As we will see with more detail in Section 6, we can mention in advance these abovementioned classes grouped 3D masks corresponding to empty regions in the original datasets. Members in these classes are precisely part of the point of discussion relative to the fact hypervoxelizations' spatial complexities make sometimes difficult their manipulation and storage: under hypervoxelization representation we are also dealing with the processing and "cargo space" of empty regions. However, we will see in next Section by considering the $n \mathrm{D}$-EVM model these regions can be taken in account in the representation without impacting spatial conciseness.

\section{The Framework $n$ D-EVM/Kohonen}

The previously defined $1 \mathrm{D}-\mathrm{KN}$ s have provided us with segmentations associated with datasets by establishing a number of classes and a size for the $(n-1) \mathrm{D}$ masks that describe the used training sets. As commented previously, each one of these $(n-1) \mathrm{D}$ masks describes in fact a subdataset by considering a hypervoxel of interest and its neighborhood of radius $r$. Then, the masks are sent as input to a $1 \mathrm{D}-\mathrm{KN}$ and through the corresponding training process there are determined their associated classes. More specifically, once the training has finished, given a $(n-1) \mathrm{D}$ mask as input it is obtained as output the number of the corresponding class which is assigned only to the input mask's central hypervoxel. Our hypervoxelizations were originally expressed with $K$ color intensities, however, we have now through a $1 \mathrm{D}-\mathrm{KN}$ these $K$ intensities have been mapped onto a set of $O$ elements: the number of classes established for training the 1DKN. Therefore, the color intensity value for a hypervoxel can now be replaced by its corresponding number of associated class given as output by the network. The hypervoxelization's original size is not affected except on its boundaries because masks of radius $r$ cannot be completed. Therefore, to consider a hypervoxelization again as the appropriate representation for storing the segmentation achieved by means of a $1 \mathrm{D}-\mathrm{KN}$ does not provides a reduction in terms of spatial complexity.

Now we reconsider our process for expressing $(n-1) \mathrm{D}$ hypervoxelizations using the $n \mathrm{D}-\mathrm{EVM}$ which was described in Section 3. We recall that given a hypervoxel with color intensity $c$ it was obtained a $n \mathrm{D}$ hyperprism whose inferior base contains vertices with the same coordinates just as the vertices in the original $(n-1) \mathrm{D}$ hypervoxel but incorporating a $n$th coordinate with zero value. On the other side, vertices in the upper base also corresponded with vertices in the original hypervoxel, but in this case, the $n$th coordinate has value $c+1$. Our proposed Framework $n \mathrm{D}-\mathrm{EVM} /$ Kohonen takes place when a dataset's segmentation achieved by a $1 \mathrm{D}-\mathrm{KN}$ is represented by using the $n \mathrm{D}-\mathrm{EVM}$, considering the conversion methodology abovementioned but replacing color intensity values by the class numbers generated as output by the corresponding $1 \mathrm{D}-\mathrm{KN}$. From a " $n$-Dimensional point of view", the $X_{n}$-axis now is referring to the number of class which is associated a hypervoxel respect to the segmented dataset.

Summarizing, the following is the workflow for our Framework $n$ D-EVM/Kohonen.

Input. A $(n-1) \mathrm{D}$ hypervoxelization under $K$ color intensities, $n \geq 2, K \geq 3$.

Step 1. Given value $r$ for radius of $(n-1) \mathrm{D}$ masks it is built a training set.

Step 2. Given values $T$ and $O$ for number of presentations and number of classes, respectively, it is trained a $1 \mathrm{D}-\mathrm{KN}$ using as training set all the masks generated in the previous step.

Step 3. The training set is used again as input for the 1D$\mathrm{KN}$ with adjusted weights. Each $(n-1) \mathrm{D}$ mask is sent as input to the $1 \mathrm{D}-\mathrm{KN}$ and it is obtained the corresponding output: the number of its associated class. This number is assigned only to the input mask's central hypervoxel (The remaining hypervoxels are classified in time when they take the role of central hypervoxels in their corresponding masks). Coordinates, respect to the original hypervoxelization, of the central hypervoxel in the input $(n-1) \mathrm{D}$ mask, are used together with its class number for building a $n \mathrm{D}$ hyperprism.

Step 4. All generated hyperprisms are integrated in such way it is obtained the corresponding whole $n \mathrm{D}-\mathrm{OPP}$ which in turn is expressed in the $n \mathrm{D}-\mathrm{EVM}$.

\section{Output. The final $n \mathrm{D}$-EVM.}

Just in the way we proceed in Section 3, Tables 10 to 14 show some $3 \mathrm{D}$ couplets, perpendicular to $X_{4}$-axis, obtained from our considered 5 datasets, Aneurism, CT-Monkey, Statue Leg, VL-Baby, and VL-CT-Knee, respectively, after the application of Framework $n \mathrm{D}-\mathrm{EVM} /$ Kohonen. The 3DEVM visualizations from these Tables were again achieved by means of visualization software developed in [18-20]. We remember the projection of $3 \mathrm{D}$ couplets under our $4 \mathrm{D}-\mathrm{EVM}$ representation collect those voxels that were grouped inside the same class by the corresponding $1 \mathrm{D}-\mathrm{KN}$. We can see, for 
TABLE 9: Classification of 3D masks according to 1D-KNs with 125 inputs, 40 output neurons, and 10 presentations.

\begin{tabular}{|c|c|c|c|c|c|}
\hline Class & Aneurism & Statue Leg & CT Monkey & VL-CT-Knee & $V L-B a b y$ \\
\hline 1 & 3,128 & 5,789 & 8 & 912,693 & 15,469 \\
\hline 2 & 21,773 & 6,349 & 16,683 & 117,273 & 8,358 \\
\hline 3 & 4,862 & 33,243 & 25,979 & $1,901,801$ & 25,431 \\
\hline 4 & $15,758,840$ & 29,837 & 31,162 & 3,150 & 58,846 \\
\hline 5 & 18,296 & $\mathbf{0}$ & 13,020 & 3,261 & 1,332 \\
\hline 6 & 5,927 & $\mathbf{0}$ & 14 & 87,610 & $1,139,659$ \\
\hline 7 & 2,231 & 15,417 & 9,116 & 15,387 & 10,155 \\
\hline 8 & 3,041 & 128,851 & 6,518 & 4,197 & 3,700 \\
\hline 9 & 8,320 & 0 & 8,784 & $8,652,128$ & $4,009,622$ \\
\hline 10 & 4,753 & 19,665 & 13,841 & 25,555 & 14,959 \\
\hline 11 & 3,037 & 13,651 & 34,950 & 435,107 & 13,488 \\
\hline 12 & 3,564 & 20,424 & 4,360 & 35,484 & 60,688 \\
\hline 13 & 8,821 & 25,873 & 2,723 & 2,005 & 4,712 \\
\hline 14 & 24,639 & 15,408 & $\mathbf{0}$ & 1,891 & 2,210 \\
\hline 15 & 904 & 0 & 91,616 & 30,631 & 23,891 \\
\hline 16 & 3,251 & 51,575 & 106,880 & 2,097 & 41,951 \\
\hline 17 & 2,377 & 16,316 & 31,121 & 115,289 & 5,112 \\
\hline 18 & 101 & 0 & 111,560 & 1,096 & 29,300 \\
\hline 19 & 2,610 & 34,834 & 6,353 & 7,794 & 113,790 \\
\hline 20 & 3,947 & 0 & 10,229 & 41,661 & 56,087 \\
\hline 21 & 9,784 & 17,119 & 3,069 & 25,514 & 3,810 \\
\hline 22 & 2,762 & 10,638 & 6,144 & 32,693 & 3,229 \\
\hline 23 & 3,142 & 45,111 & 258 & 31,775 & 6,435 \\
\hline 24 & 18,959 & $9,473,821$ & 45 & 6,852 & 21,690 \\
\hline 25 & 5,640 & 9,689 & 10,385 & 2,035 & 33,498 \\
\hline 26 & 3,089 & 0 & 4,694 & 68,408 & 1,323 \\
\hline 27 & 930 & $\mathbf{0}$ & $3,009,111$ & $12,234,834$ & 49,447 \\
\hline 28 & 5,513 & 0 & 17 & 25,474 & 17,353 \\
\hline 29 & 5,496 & 11,810 & 7,282 & 34,373 & 23,895 \\
\hline 30 & 7,333 & 0 & 1 & 2,489 & 10,582 \\
\hline 31 & 5,372 & 0 & 8,586 & 22,581 & 3,057 \\
\hline 32 & 4,885 & 0 & 61 & 138,980 & 9,024 \\
\hline 33 & 12,722 & 8,559 & 19,599 & 18,576 & 3,405 \\
\hline 34 & 6,908 & 0 & 59,738 & 1,315 & 27,012 \\
\hline 35 & 1,234 & $\mathbf{0}$ & 1,906 & 20,623 & 4,184 \\
\hline 36 & 117 & 0 & 5 & 29,828 & 20,019 \\
\hline 37 & 8,460 & 113,662 & 6,703 & 78,950 & 32,802 \\
\hline 38 & 5,258 & 0 & 5,623 & 8,005 & 3,350 \\
\hline 39 & 5,368 & $\mathbf{0}$ & 6,365 & 127,575 & 6,370 \\
\hline 40 & 5,614 & 0 & 8,723 & 89,885 & 50,131 \\
\hline
\end{tabular}

example, in Table 13, how it is possible to appreciate couplets 12,19 , and 28 were bone tissue is only present. On the other hand, 3D couplets $5,17,20$, and 26 describe the grouping of skin tissue (and other support medical accessories). A very similar situation can be appreciated respect to voxelization VL-CT-Knee (Table 14) where all of the shown 3D couplets, except 12,21 , and 28, correspond to different parts of bone tissue.

In Section 5 we made mention of the fact that all considered $1 \mathrm{D}$-KNs were grouping more than $45 \%$ of the elements in their training sets in just one class. This can be visually appreciated in 3D couplets 5, 27, 19, 10, and 28 from Tables 10, $11,12,13$, and 14 , respectively. All of these $3 \mathrm{D}$ couplets present a block-like structure whose minimal bounding box has the same dimensions than the original voxelizations minus the volume removed by those $3 \mathrm{D}$ masks of radius 2 that could not be completed at the datasets' boundaries. These are the regions that correspond to empty space in the original voxelizations. In Section 5 we gave an argument regarding the observation that these $3 \mathrm{D}$ couplets are considered in 
TABLE 10: Some 3D couplets, perpendicular to color axis, extracted from the 4D-EVM produced by means of Framework $n \mathrm{D}$-EVM/Kohonen and associated to dataset Aneurism.

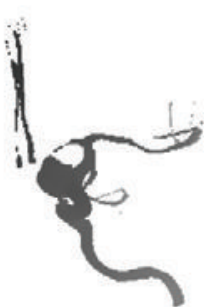

3D Couplet 3

CCL_EVM ${ }_{3}\left(\pi_{4}\left(\Phi_{3}^{4}\right)\right)=58$

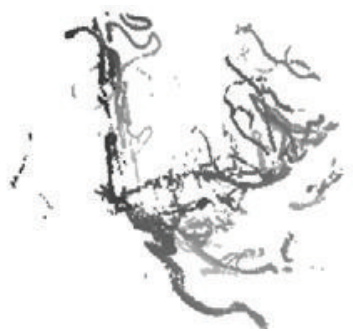

3D Couplet 15

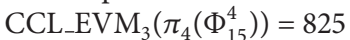

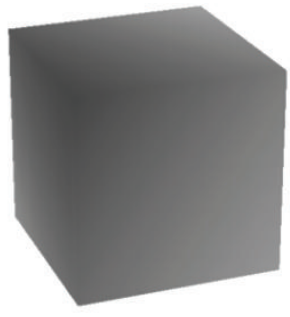

3D Couplet 5

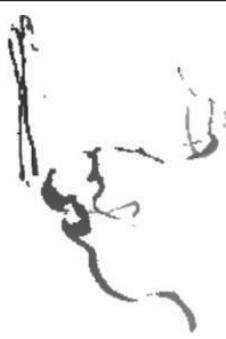

3D Couplet 7

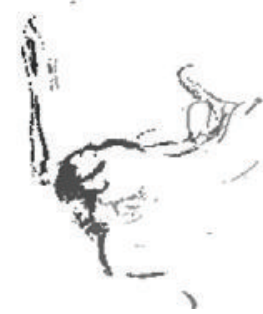

3D Couplet 12 $\mathrm{CCL}_{\mathrm{EVM}}\left(\pi_{4}\left(\Phi_{5}^{4}\right)\right)=78$ $\mathrm{CCL}_{2} \mathrm{EVM}_{3}\left(\pi_{4}\left(\Phi_{7}^{4}\right)\right)=87$

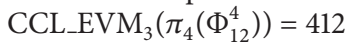

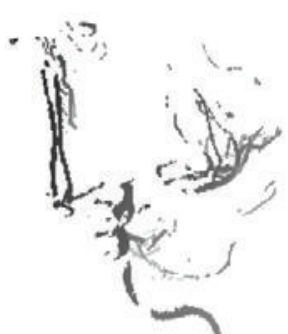

3D Couplet 30

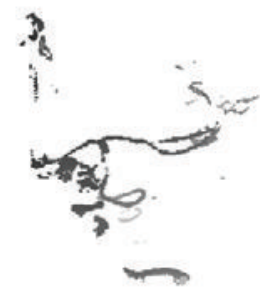

3D Couplet 39 $\mathrm{CCL}_{2} \mathrm{EVM}_{3}\left(\pi_{4}\left(\Phi_{30}^{4}\right)\right)=508$

$\mathrm{CCL} \_E V M_{3}\left(\pi_{4}\left(\Phi_{39}^{4}\right)\right)=239$

TABLE 11: Some 3D couplets, perpendicular to color axis, extracted from the 4D-EVM produced by means of Framework $n \mathrm{D}$-EVM/Kohonen and associated to dataset CTMonkey.

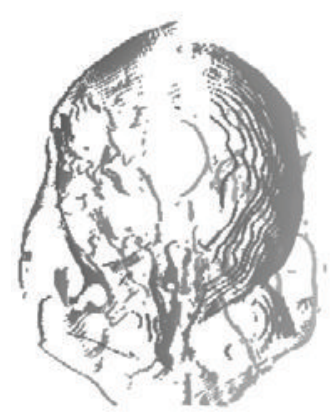

3D Couplet 3 CCL_EVM ${ }_{3}\left(\pi_{4}\left(\Phi_{3}^{4}\right)\right)=1,554$

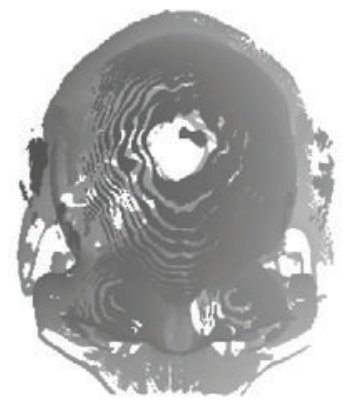

3D Couplet 18 CCL_EVM $\operatorname{EVM}_{3}\left(\pi_{4}\left(\Phi_{18}^{4}\right)\right)=1,169$

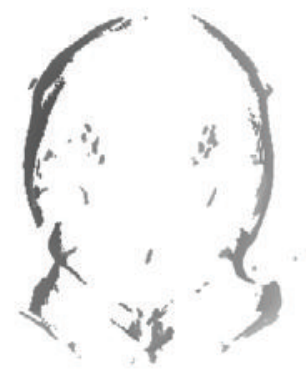

3D Couplet 6 $\mathrm{CCL}_{\mathrm{EVM}}\left(\pi_{4}\left(\Phi_{6}^{4}\right)\right)=402$

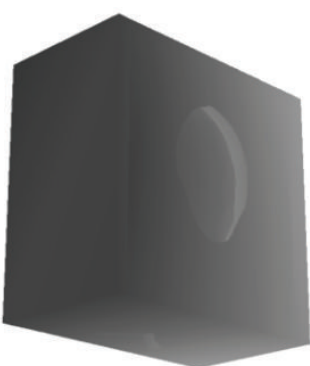

3D Couplet 27

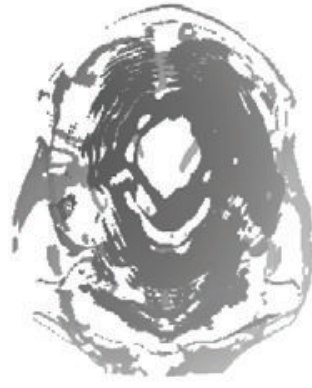

3D Couplet 12 $\mathrm{CCL}_{2} \mathrm{EVM}_{3}\left(\pi_{4}\left(\Phi_{12}^{4}\right)\right)=551$

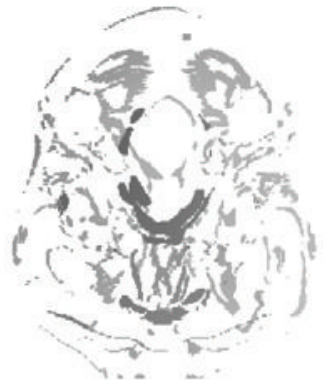

3D Couplet 31 CCL_EVM ${ }_{3}\left(\pi_{4}\left(\Phi_{31}^{4}\right)\right)=650$

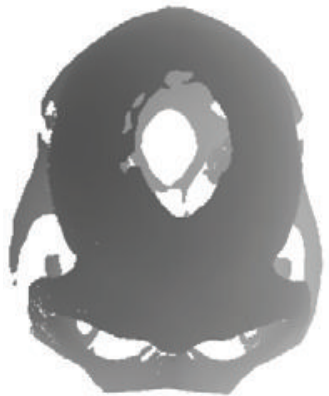

3D Couplet 15 CCL_EVM ${ }_{3}\left(\pi_{4}\left(\Phi_{15}^{4}\right)\right)=581$

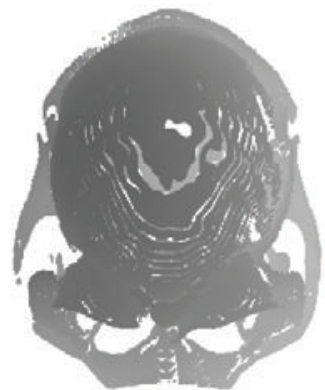

3D Couplet 34 $\operatorname{CCL}_{\operatorname{EVM}}\left(\pi_{4}\left(\Phi_{34}^{4}\right)\right)=1,039$ 
TABLE 12: Some 3D couplets, perpendicular to color axis, extracted from the 4D-EVM produced by means of Framework $n \mathrm{D}$-EVM/Kohonen and associated to dataset Statue Leg.

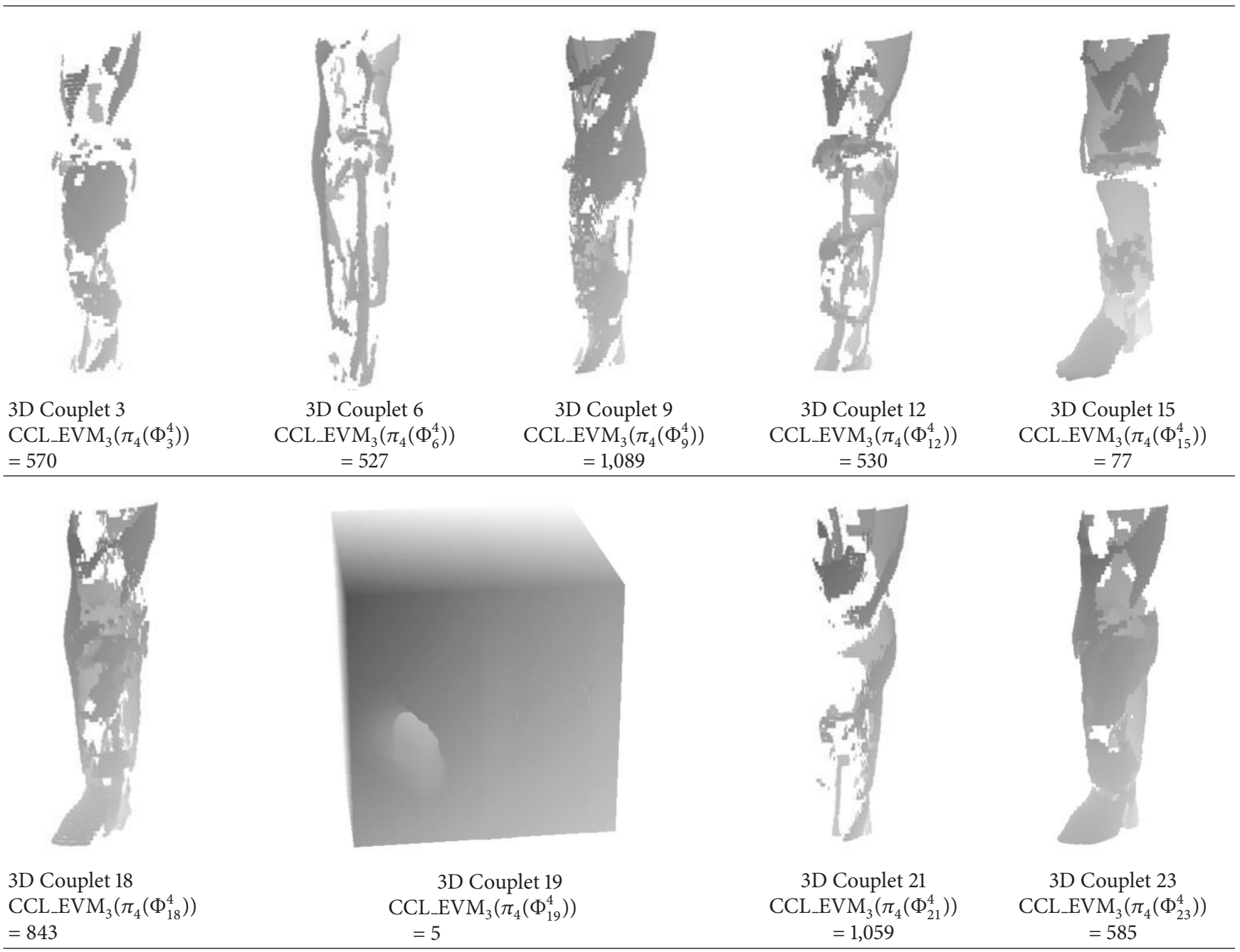

our 4D-EVM representation. We also mentioned this empty space is precisely one of the disadvantages behind a voxelization because it only contributes to spatial complexity. Our argument serves for the purpose of touching a crucial point: the spatial complexity of the representations achieved by the Framework $n \mathrm{D}$-EVM/Kohonen. Table 15 shows the cardinalities for the $4 \mathrm{D}$-EVMs obtained for expressing our 5 voxelizations. We also compute (14) in order to observe the number of times our 4D models are exceeded by its original voxelizations. We can see for example, dataset $C T$ Monkey originally required to be stored in more than 4 million voxels. In Section 3 we saw this dataset had a direct 4D-EVM representation with 3,573,172 extreme vertices: an slight improvement over the original voxelization. By taking in account the proposed framework we have were used 789,320 extreme vertices, or in other words, the original voxelization is 5.1477 times greater, in terms of spatial storage, than the final 4D-EVM's cardinality. A more impacting example comes from dataset Aneurism: the original voxelization is formed by $16,777,216$ voxels; the direct $4 \mathrm{D}$-EVM representation had 1,317,008 extreme vertices; and finally, the Framework $n$ D-EVM/Kohonen shared a 4D-EVM with 517,318 extreme vertices: giving the ratio 32.4311 . We should take in account our representations are also considering the storing of the regions corresponding to empty space. This give us an advantage by storing in the same representation both the occupied and the empty space but without affecting the memory needed to store the models. These results share us evidence of the conciseness' power achieved when the grouping properties of $1 \mathrm{D}-\mathrm{KN}$ s are combined with the storing properties of a model like the $n \mathrm{D}$-EVM: spatial complexity is reduced and a more appropriate segmentation is achieved, which impacts how the elements in the datasets are classified and by consequence accessed. For example, Table 16 shows some $2 \mathrm{D}$ sections corresponding to one of the 3D couplets from the model CT-Monkey after the application of Framework $n \mathrm{D}-\mathrm{EVM} /$ Kohonen. There are shown, via direct application of Algorithm 4, 17 of these $2 \mathrm{D}$ sections which allow understand the internal organization of the selected $3 \mathrm{D}$ couplet. It is possible to visualize some internal bone structures associated to the monkey's cranium. 
TABLE 13: Some 3D couplets, perpendicular to color axis, extracted from the 4D-EVM produced by means of Framework $n \mathrm{D}$-EVM/Kohonen and associated to dataset $V L-B a b y$.

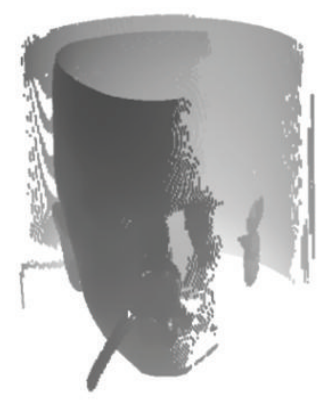

3D Couplet 5

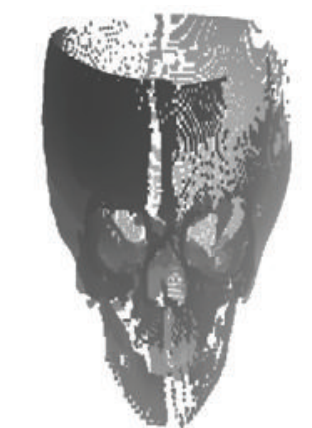

3D Couplet 19

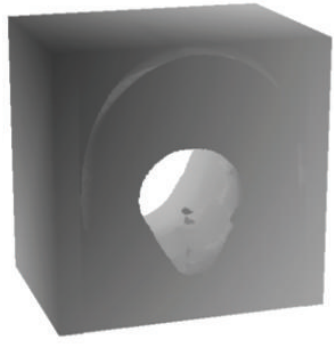

3D Couplet 10

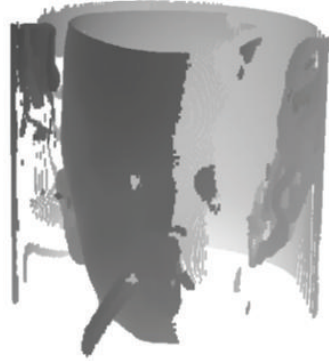

3D Couplet 20

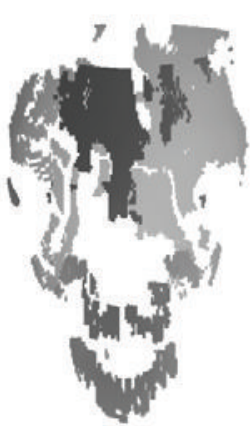

3D Couplet 12

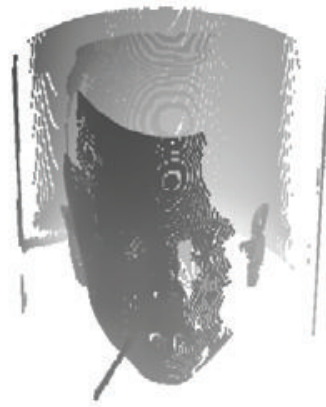

3D Couplet 17

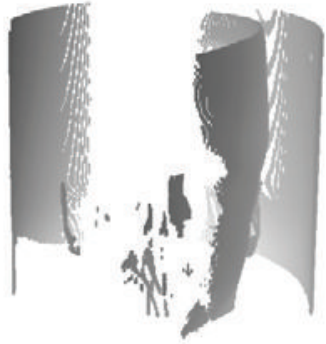

3D Couplet 26

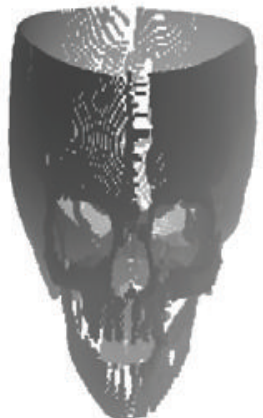

3D Couplet 28

\section{Quality of Segmentations Achieved by the $n$ D-EVM/Kohonen Framework}

In some sense, we have sustained, in implicit way, datasets' representations generated by the Framework $n \mathrm{D}-\mathrm{EVM} /$ Kohonen provide appropriate segmentations that are in fact better than those segmentations produced by simple and direct classification based only in color intensities. Part of the asseveration is sustained in the sense our 1D-KNs classifications are taking in account geometry and topology surrounding each hypervoxel in the dataset: the concept of multidimensional mask as training element. This Section provides more formal arguments to sustain the segmentations that have been produced have good quality and they are even better than those produced by classifications based only in color intensities. For this purpose, some previously defined concepts are revisited and error functions are introduced.

Original 4D-EVM representations for datasets Aneurism, CT-Monkey, and Statue Leg were described in Section 3 (Tables 3, 4, and 5). The respective visual comparison with Tables 10, 11, and 12 clearly show us how our proposed framework has provided more cohesive representations for these voxelizations. We sustain our claim by considering the number of produced 3D CCs. Let function $\mathrm{CCL}_{3}$ be defined as follows:

$$
\mathrm{CCL}_{3}(p)=\sum_{i=1}^{n p_{4}} \operatorname{CCL}_{-} \operatorname{EVM}_{3}\left(\pi_{4}\left(\Phi_{i}^{4}(p)\right)\right)
$$

where $n p_{4}$ denotes the number of $3 \mathrm{D}$ couplets perpendicular to $X_{4}$-axis for $4 \mathrm{D}$-OPP $p$ expressed under the EVM, and function CCL_EVM $\mathrm{EM}_{3}$, originally defined in Section 3, shares the number of 3D CCs for a 3D-OPP, a 3D couplet in the current instance. Then, it is clear $\mathrm{CCL}_{3}$ shares us the total number of $3 \mathrm{D}$ CCs present in all couplets, perpendicular to $X_{4}$-axis, associated to the input $4 \mathrm{D}-\mathrm{EVM} p$. We recall CCs computation can be achieved by our previously mentioned CCLEVM-Based algorithm whose specific details are presented in [21]. We compute $\mathrm{CCL}_{3}$ for representations of datasets Aneurism, Statue Leg, and CT-Monkey. Table 17 presents the corresponding values for both direct 4D-EVM representation (Section 3) and under Framework $n \mathrm{D}-\mathrm{EVM} / \mathrm{Kohonen.} \mathrm{The}$ same source data were used for building both representations, but as seen in Table 17, our framework produces much more cohesive and compact representations. A counterargument to this claim could arise by establishing the gained compaction and cohesion is product of the fact our 1D-KNs "forces" the data to be classified under 40 classes while the segmentation based on intensity has "more space" because 256 classes are available. We attack this situation by proposing some error functions. Let vector $\left[\begin{array}{llll}g_{1, i, j} & g_{2, i, j} & \cdots & g_{\psi, i, j}\end{array}\right]^{T}$ be a linearized $(n-1) \mathrm{D}$ mask in a training set TS, such that it was characterized as part of class $\kappa_{j}$ once a $1 \mathrm{D}-\mathrm{KN}$ was trained precisely with TS. Index $i$ only refers to an arbitrary position assigned to the mask belonging class $\kappa_{j}$. Now consider to Weights Vector $W_{j}=\left[\begin{array}{llll}W_{1, j} & W_{2, j} & \cdots & W_{\psi, j}\end{array}\right]^{T}$ associated to class $\kappa_{j} . W_{j}$ can be also seen as a linearized $(n-1) \mathrm{D}$ mask and for instance it can be also compared with those $(n-1) \mathrm{D}$ masks 
TABLE 14: Some 3D couplets, perpendicular to color axis, extracted from the 4D-EVM produced by means of Framework $n \mathrm{D}$-EVM/Kohonen and associated to dataset VL-CT-Knee.
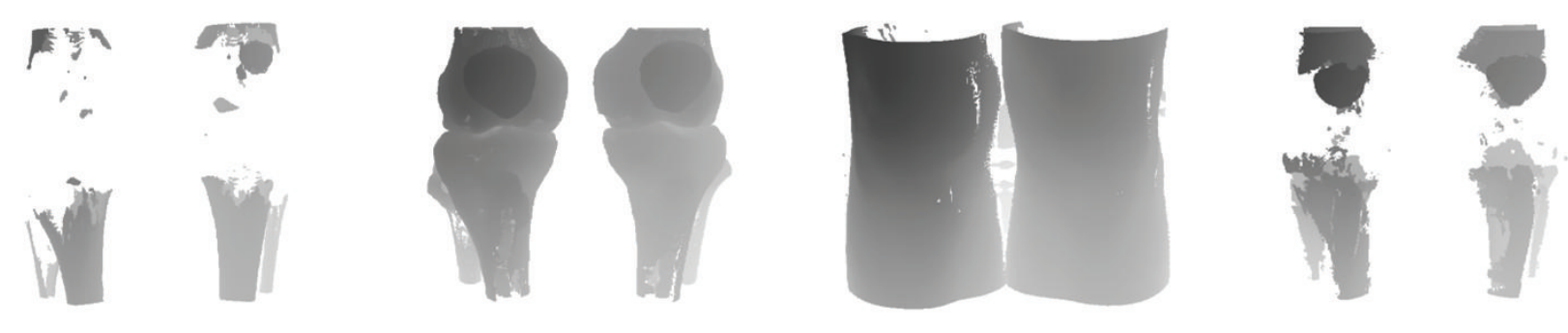

3D Couplet 3

3D Couplet 4

3D Couplet 12

3D Couplet 18

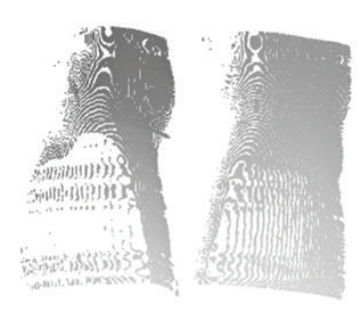

3D Couplet 21
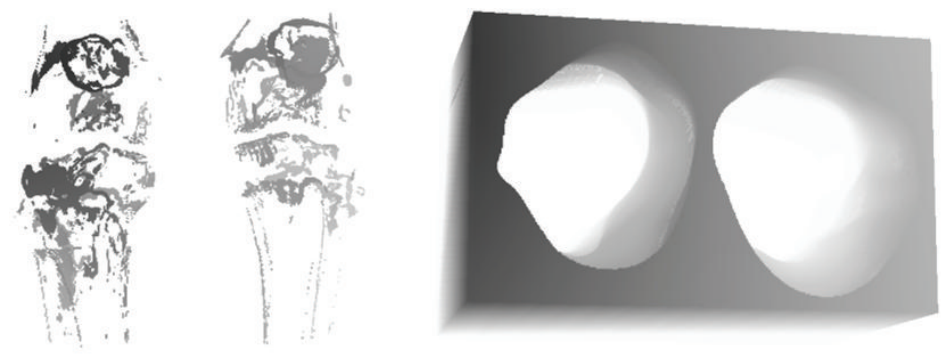

3D Couplet 23

3D Couplet 28
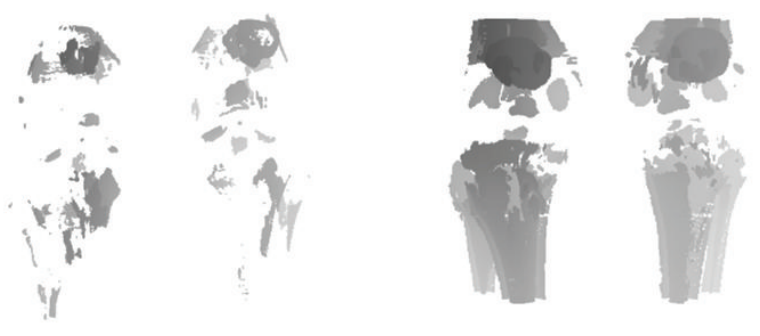

3D Couplet 30

3D Couplet 33

TABLE 15: The ratio number of voxels/number-of-extreme-vertices for datasets shown in Tables 1 and 7 after processing via Framework $n \mathrm{D}$ EVM/Kohonen.

\begin{tabular}{lccc}
\hline Object $p$ & $\begin{array}{c}\text { Voxelization Size } \\
(\text { number of voxels })\end{array}$ & $\begin{array}{c}\text { Card }\left(\mathrm{EVM}_{4}(p)\right) \text { by } \\
\text { Framework } n \text { D-EVM/Kohonen }\end{array}$ & $\frac{x_{1} \operatorname{Size} \cdot x_{2} \operatorname{Size} \cdot x_{3} \operatorname{Size}}{\left.\mathrm{Card}_{(\mathrm{EVM}}(p)\right)}$ \\
\hline Aneurism & $16,777,216$ & 517,318 & 32.4311 \\
Statue Leg & $10,814,133$ & 604,970 & 17.8754 \\
CT Monkey & $4,063,232$ & 789,320 & 5.1477 \\
VL-CT-Knee & $26,471,255$ & $2,799,470$ & 9.4558 \\
VL-Baby & $6,422,528$ & $1,136,792$ & 5.6496 \\
\hline
\end{tabular}

located in the corresponding class [34]. We define then to the "Error in Class j respect to $W_{j}$ ", denoted by $\operatorname{Err}_{j}^{W}$, as [34]

$$
\mathrm{ERR}_{j}^{W}=\sum_{i=1}^{\operatorname{Card}\left(\kappa_{j}\right)}\left(\sum_{p=1}^{\psi}\left|g_{p, i, j}-W_{p, j}\right|\right) .
$$

It is clear by computing $\mathrm{ERR}_{j}^{W}$ we are obtaining the differences existing between each $(n-1) \mathrm{D}$ mask that belongs to class $j$ with Weights Vector $W_{j}$, which in fact is its representative. We can think if $\mathrm{ERR}_{j}^{W}$ has a low value then $W_{j}$ is a good representative for all the $(n-1) \mathrm{D}$ masks belonging to $\kappa_{j}$. This reasoning can be extended for considering all the representatives of all classes for a given $1 \mathrm{D}-\mathrm{KN}$. Therefore, we have a value for measuring the classification quality shared by a network. We say the "Global Error for Training Set TS respect to its Representatives", denoted by $\mathrm{ERR}_{\mathrm{TS}}^{W}$, can be defined as [34]

$$
\mathrm{ERR}_{\mathrm{TS}}^{W}=\sum_{j=1}^{O} \mathrm{ERR}_{j}^{W}
$$

Prior to make a comparison between the two presented 4D representations by using the above error functions we face 
TABLE 16: Visualizing some 2D sections perpendicular to $X_{4}$-axis which were processed from one $3 \mathrm{D}$ couplet of the $4 \mathrm{D}$-OPP associated to dataset CTMonkey after the application of Framework $n \mathrm{D}$-EVM/Kohonen.

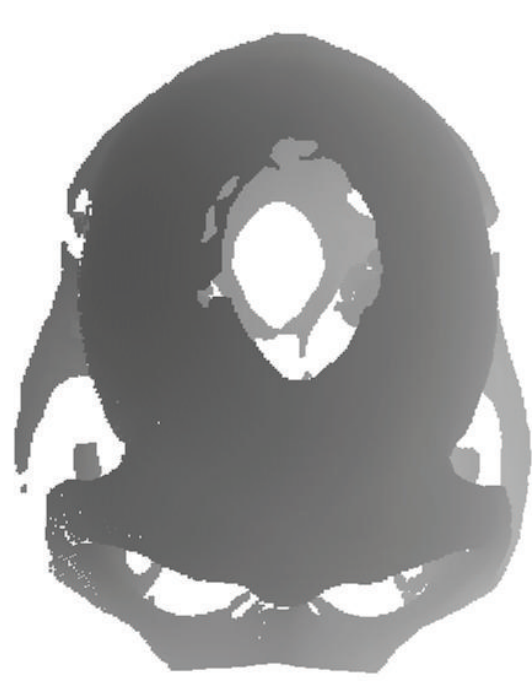

3D Couplet 15

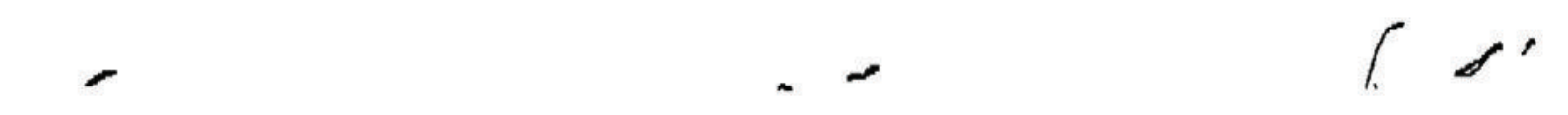

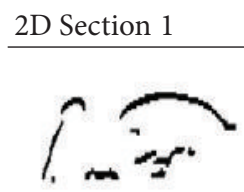

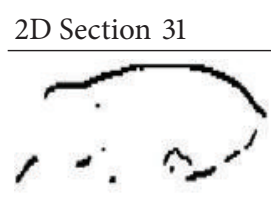

2D Section 71

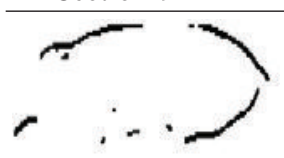

2D Section 131

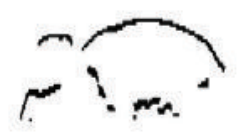

2D Section 171
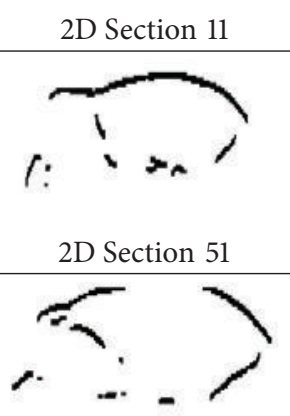

2D Section 91

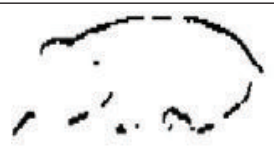

2D Section 141

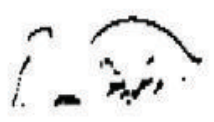

2D Section 181

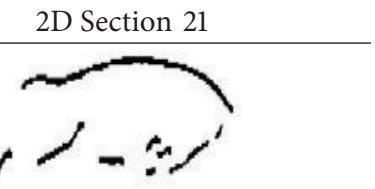

2D Section 61

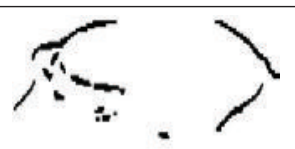

2D Section 111

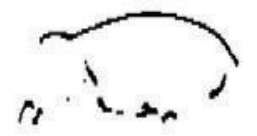

2D Section 161

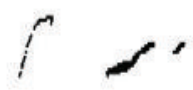

2D Section 191 
TABLE 17: $\mathrm{CCL}_{3} \mathrm{~s}^{\prime}$ comparisons for representation of volume datasets (see text for details).

\begin{tabular}{lcc}
\hline Dataset & $\begin{array}{c}\mathrm{CCL}_{3} \text { under direct } \\
\text { 4D-EVM } \\
\text { representation }\end{array}$ & $\begin{array}{c}\mathrm{CCL}_{3} \text { under } \\
\text { Framework } \\
n \mathrm{D} \text {-EVM/Kohonen }\end{array}$ \\
\hline Aneurism & 130,483 & 21,403 \\
Statue Leg & 778,137 & 17,280 \\
CTMonkey & 431,651 & 23,352 \\
\hline
\end{tabular}

the situation related to the fact that segmentation based on intensities only considers the color intensity of the hypervoxel of interest and nothing more; while our Framework $n \mathrm{D}-\mathrm{EVM} /$ Kohonen requires, as specified in Section 5, the creation of a training set whose elements are $(n-1) \mathrm{D}$ masks which contain the hypervoxels of interest and also their neighborhoods of radius $r$. This situation recall us we are considering different aspects for the considered segmentations, however, the fundament behind the use of $(n-1) \mathrm{D}$ masks is to take in account geometrical and topological properties such that a $1 \mathrm{D}-\mathrm{KN}$ use them in order to achieve and to enhance the classification. On the other hand, because of simplicity in implementing color-intensity based segmentation the notion of a representative for a class is absent: the hypervoxels included in a class share the same intensity. In order to apply our proposed error functions to classification achieved only by color intensity we build a representative for elements in a same class. Consider a hypervoxel $v$ with color intensity $c+1$, then, it is located in class $\kappa_{c+1}$. Now, we will consider the neighborhood of radius $r$ for the given hypervoxel $v$ respect to the original hypervoxelization. Then, we are defining a $(n-1) \mathrm{D}$ mask whose center is $v$. Given value for $r$, the corresponding $(n-$ 1)D mask also have $(2 r+1)^{(n-1)}$ elements (Equation (17)). The mask can be also linearized in such way we obtain the vector representation $\left[\begin{array}{lllll}g_{1, i, c+1} & g_{2, i, c+1} & \cdots & g_{\psi, i, c+1}\end{array}\right]^{T}$. Index $i$ only refers to an arbitrary position assigned to the mask belonging class $\kappa_{c+1}$. One of the elements in the vector is precisely color intensity, $c+1$, of central hypervoxel $v$ while the rest are the color intensities of the hypervoxels inside its neighborhood. Given the above elements we build a representative of class $\kappa_{c+1}$, but under color-intensity based segmentation (CIBS), as follows:

$$
W_{c+1}^{\mathrm{CIBS}}=\frac{1}{\operatorname{Card}\left(\kappa_{c+1}\right)}\left[\begin{array}{c}
\sum_{i=1}^{\operatorname{Card}\left(\kappa_{c+1}\right)} g_{1, i, c+1} \\
\sum_{i=1}^{\operatorname{Card}\left(\kappa_{c+1}\right)} g_{2, i, c+1} \\
\vdots \\
\sum_{i=1}^{\operatorname{Card}\left(\kappa_{c+1}\right)} g_{\psi, i, c+1}
\end{array}\right] .
$$

That is, given the members of class $\kappa_{c+1}$, under color-intensity based segmentation, its representative has as components the average color intensity for each one of the $(2 r+1)^{(n-1)}$
TABLE 18: Global errors' comparisons for representation of volume datasets (see text for details).

\begin{tabular}{lcc}
\hline Dataset & $\begin{array}{c}\mathrm{ERR}_{\mathrm{TS}}^{W} \text { under direct } \\
\text { 4D-EVM } \\
\text { representation }\end{array}$ & $\begin{array}{c}\mathrm{ERR}_{\mathrm{TS}}^{W} \text { under } \\
\text { Framework } \\
n \mathrm{D}-\mathrm{EVM} / \text { Kohonen }\end{array}$ \\
\hline Aneurism & $8,707,543.1607$ & $5,484,105.9349$ \\
Statue Leg & $24,690,070.9348$ & $11,097,469.3973$ \\
CTMonkey & $17,304,147.229$ & $10,575,227.2008$ \\
\hline
\end{tabular}

positions in each one of the $(n-1) \mathrm{D}$ masks defined for the class' members. On one side, we have now representatives in order to apply them over our proposed error functions. On the other side, we proceeded in the way described because if it is false our asseveration related to the fact geometry and topology described by a neighborhood enhances classification, in particular with our $1 \mathrm{D}-\mathrm{KNs}$, then the masks and representatives defined by use of (22) when applied over our error functions will report results as good, and even better, than those reported by the use of $1 \mathrm{D}-\mathrm{KN}$. Table 18 shows the global error respect to its representatives, obtained for each one of the training sets generated with datasets Aneurism, Statue Leg and CT-Monkey. The results presented are encouraging: global errors under direct 4D-EVM representation are $1.5877,2.2248$, and 1.6362 times greater than those obtained by Framework $n$ D-EVM/Kohonen for our datasets Aneurism, Statue Leg and CT-Monkey, respectively. We then recur again to Table 17 where $\mathrm{CCL}_{3}$ s under direct $4 \mathrm{D}$-EVM representation are 6.0964, 45.031, and 18.4845 times greater than those generated by Framework $n \mathrm{D}$-EVM/Kohonen for the same datasets, respectively. This experimental analysis has share us elements to sustain cohesiveness produced by our proposal is appropriate because presented measures tell us the voxels were better positioned by the $1 \mathrm{D}-\mathrm{KN}$ s producing better segmentation based in information supported by $(n-1) \mathrm{D}$ neighborhoods.

\section{Conclusions and Future Work}

In this work we have developed the Framework $n \mathrm{D}$ EVM/Kohonen whose purposes are (1) the concise representation of multidimensional datasets, and (2) a modeling of the data they contain in such way the information contained can be accessed in efficient and useful way. These purposes have been achieved by the consideration of the Extreme Vertices Model in the $n$-Dimensional Space as the representation scheme that provides conciseness, as seen in Sections 3 and 6, but providing an appropriate organization of datasets' elements by means of taking in account an extra geometrical dimension whose objective is allow grouping only those elements characterized as members of the same class. Precisely, the classification process, is achieved by the use of 1-Dimensional Kohonen Networks whose task is the automatic nonsupervised characterization of the elements in a dataset. As seen in Section 5, we have defined training sets taking in account the geometry and topology surrounding each hypervoxel. The experimental results we have obtained show us the use of $1 \mathrm{D}$-KNs trained according to our proposal 
produce classes whose members form connected components with more cohesion and more compact than those generated by segmentation achieved only by color intensity. The expressed asseverations, discussed in Section 7, have been sustained by the use of function errors that have shown, for our study cases, that classification achieved by means of $1 \mathrm{D}-\mathrm{KN}$ s is a good option due to the way members in each class relate to their corresponding representatives. Moreover, we have seen these $1 \mathrm{D}-\mathrm{KN}$-assisted classifications impact even more the conciseness of the $n \mathrm{D}$-EVMs obtained producing compression ratios, for our study cases, between 5.1477 and 32.4311. Summarizing, we have presented evidence related to how our established framework effectively combine the power of the $n \mathrm{D}-\mathrm{EVM}$ and the $1 \mathrm{D}-\mathrm{KN}$ in order to produce very concise datasets' representations but also providing an appropriate segmentation.

In terms of lines of future research we make mention we will direct our efforts into the manipulation of datasets where hypervoxels have associated, besides spatial information, data that requires to be expressed with more than a scalar value. In fact, an immediate line of attack could be the manipulation of datasets in RGB colorspace where precisely the red, green and blue components are separated and forming a vector. We hypothesize the Framework $n \mathrm{D}-\mathrm{EVM}$ /Kohonen could be able to manipulate and represent this kind of situation in such way not only one additional dimension is required, maybe more extra dimensions are going to be taken in account.

We also expect to aboard in the future the study of the utility of the classifications produced by $1 \mathrm{D}-\mathrm{KNs}$ in terms of application in specific areas and by taking in account evaluation by experts. More specifically, we are going to attack the particular topic of medical datasets' segmentations. The idea is to represent and manipulate these datasets using our Framework $n$ D-EVM/Kohonen but we will analyze particularly the automatic tissue classification task. In a first stage, classification is going to be automatically achieved by our $1 \mathrm{D}-\mathrm{KN}$ and it will be evaluated against segmentations produced by physicians. In a second stage there will be studied evaluations' results and procedures, if it would be necessary, for enhancing them. In this sense we can mention some experiments have been developed, in [31, 34, 35], for automatically classifying brain tissue in Computed Tomography Brain Slices. We can mention the obtained results are encouraging. Such as in our current line of research, we aim to propose concise and useful representations for these medical datasets but without compromising the information they contain because of its obvious importance and relevance. Finally, in a more independent-of-the-application point of view, we are also going to study algorithmic approaches in order to automatically avoid the phenomenon of under and over segmentation and the way these methodologies can be incorporated in the Framework $n \mathrm{D}-\mathrm{EVM}$ /Kohonen.

\section{Conflict of Interests}

The authors declare that there is no conflict of interests regarding the publication of this paper.

\section{References}

[1] A. Jonas and N. Kiryati, "Digital representation schemes for 3-D curves," Tech. Rep. CC PUB \#114, The Technion-Israel Institute of Technology, Haifa, Israel, 1995.

[2] G. Klajnsek, B. Rupnik, and D. Spelic, "An improved quadtreebased algorithm for lossless compression of volumetric datasets," in Proceedings of the 6th WSEAS Inernational Conference on Computational Intelligence, Man-Machine Systems and Cybernetics, vol. 1, pp. 264-270, Tenerife, Spain, December 2007.

[3] W. Song, S. Hua, Z. Ou, H. An, and K. Song, "Octree based representation and volume rendering of three-dimensional medical data sets," in Proceedings of the International Conference on BioMedical Engineering and Informatics (BMEI '08), vol. 1, pp. 316-320, May 2008.

[4] R. Pérez-Aguila, "Modeling and manipulating 3D datasets through the extreme vertices model in the $\mathrm{n}$-dimensional space (nD-EVM)," Research in Computer Science, vol. 31, pp. 15-24, 2007.

[5] N. Torbati, A. Ayatollahi, and A. Kermani, "An efficient neural network based method for medical image segmentation," Computers in Biology and Medicine, vol. 44, no. 1, pp. 76-87, 2014.

[6] M. Awad, "An unsupervised artificial neural network method for satellite image segmentation," The International Arab Journal of Information Technology, vol. 7, no. 2, pp. 199-205, 2010.

[7] C. Chandhok, "A novel approach to image segmentation using artificial neural networks and k-means clustering," International Journal of Engineering Research and Applications, vol. 2, no. 3, pp. 274-279, 2012.

[8] J. Jiang, P. Trundle, and J. Ren, "Medical image analysis with artificial neural networks," Computerized Medical Imaging and Graphics, vol. 34, no. 8, pp. 617-631, 2010.

[9] A. Aguilera, Orthogonal polyhedra: study and application [Ph.D. thesis], Universitat Politècnica de Catalunya, 1998.

[10] R. Pérez-Aguila, Orthogonal polytopes: study and application [Ph.D. thesis], Universidad de las Américas Puebla, 2006, http:// catarina.udlap.mx/u_dl_a/tales/documentos/dsc/perez_a_r/.

[11] M. Spivak, Calculus on Manifolds: A Modern Approach to Classical Theorems of Advanced Calculus, HarperCollins, 1965.

[12] R. Pérez-Aguila, "Towards a new approach for modeling volume datasets based on orthogonal polytopes in four-dimensional color space," Engineering Letters, vol. 18, no. 4, pp. 326-340, 2010.

[13] H. S. M. Coxeter, Regular Polytopes, Dover, New York, NY, USA, 2nd edition, 1963.

[14] D. M. Sommerville, An Introduction to the Geometry of $N$ Dimensions, Dover, New York, NY, USA, 1958.

[15] S. Roettger, The Volume Library, Web Site, http://www9.informatik.uni-erlangen.de/External/vollib/.

[16] Department of Radiology, University of Iowa Datasets, 2009, http://www.medicine.uiowa.edu/radiology/.

[17] University of Tübingen, "The official volren and volvis homepage," 2014, http://www.gris.uni-tuebingen.de/edu/areas/scivis/ volren/software/software.html.

[18] R. Ruiz-Rodríguez, Implementación del EVM (Extreme VerticesModel) en Java [M.S. thesis], Universidad de las AméricasPuebla (UDLAP), 2002, http://catarina.udlap.mx/u_dl_a/tales/ documentos/msp/ruiz_r_r/.

[19] R. R. Rodríguez, "A 3D editor for orthogonal polyhedra based on the extreme vertices model," in Décimo Congreso Internacional de Investigación en Ciencias Computacionales (CIICC '03), pp. 968-5823, Oaxtepec, Mexico, October 2003. 
[20] R. Ruiz Rodríguez, "Using the ordered union of disjoint boxes model for the visualization as solid objects of orthogonal pseudo polyhedra defined," in II Congreso Internacional de Informática y Computación de la ANIEI (ANIEI '03), Zacatecas, México, October 2003.

[21] R. Pérez-Aguila, "Efficient boundary extraction from orthogonal pseudo-polytopes: an approach based on the $n \mathrm{D}$-EVM," Journal of Applied Mathematics, vol. 2011, Article ID 937263, 29 pages, 2011.

[22] S. Marchand-Maillet and Y. M. Sharaiha, Binary Digital Image Processing: A Discrete Approach, Academic Press, 1999.

[23] A. Rosenfeld and J. L. Pfaltz, "Sequential operations in digital picture processing," Journal of the ACM, vol. 13, no. 4, pp. 471494, 1966.

[24] R. Pérez-Aguila, "Computing the discrete compactness of orthogonal pseudo-polytopes via their $n \mathrm{D}$-EVM Representation," Mathematical Problems in Engineering, vol. 2010, Article ID 598910, 28 pages, 2010.

[25] J. Rodríguez and D. Ayala, "Erosion and Dilation on 2D and 3D digital images: a new size-independent approach," in Proceedings of the Vision Modeling and Visualization Conference (VMV '01), vol. 1, pp. 143-150, Stuttgart, Germany, November 2001.

[26] E. Davalo and P. Naïm, Neural Networks, The Macmillan Press, New York, NY, USA, 1992.

[27] H. Ritter, T. Martinetz, and K. Schulten, Neural Computation and Self-Organizing Maps: An Introduction, Addison-Wesley, New York, NY, USA, 1992.

[28] J. Hilera and V. Martínez, Artificial Neural Networks, Alfaomega, Mexico City, Mexico, 2000, (Spanish).

[29] F. Peng, K. Yuan, S. Feng, and W. Chen, "Pre-processing of CT brain images for content-based image retrieval," in Proceedings of the 1st International Conference on BioMedical Engineering and Informatics (BMEI '08), vol. 2, pp. 208-212, May 2008.

[30] M. Berthod, Z. Kato, S. Yu, and J. Zerubia, "Bayesian image classification using Markov random fields," Image and Vision Computing, vol. 14, no. 4, pp. 285-295, 1996.

[31] R. Pérez-Aguila, "Brain tissue characterization via nonsupervised one-dimensional Kohonen networks," in Proceedings of the 19th International Conference on Electronics Communications and Computers (CONIELECOMP'09), pp. 197-201, IEEE Computer Society, Puebla, México, February 2009.

[32] R. Pérez-Aguila, P. Gómez-Gil, and A. Aguilera, "Nonsupervised classification of 2D color images using kohonen networks and a novel metric," in Progress in Pattern Recognition, Image Analysis and Applications, vol. 3773 of Lecture Notes in Computer Science, pp. 271-284, Springer, Berlin, Germany, 2005.

[33] R. Pérez-Aguila, An Introduction to Artificial Neural Computing, El Cid Editor, 1st edition, 2012, (Spanish).

[34] R. Pérez-Aguila, "Enhancing brain tissue segmentation and image classification via $1 \mathrm{D}$ Kohonen Networks and discrete compactness: an experimental study," Engineering Letters, vol. 21, no. 4, pp. 171-180, 2013.

[35] R. Pérez-Aguila, "Novel discrete compactness-based training for vector quantization networks: enhancing automatic brain tissue classification," Advances in Artificial Neural Systems, vol. 2013, Article ID 278241, 18 pages, 2013. 

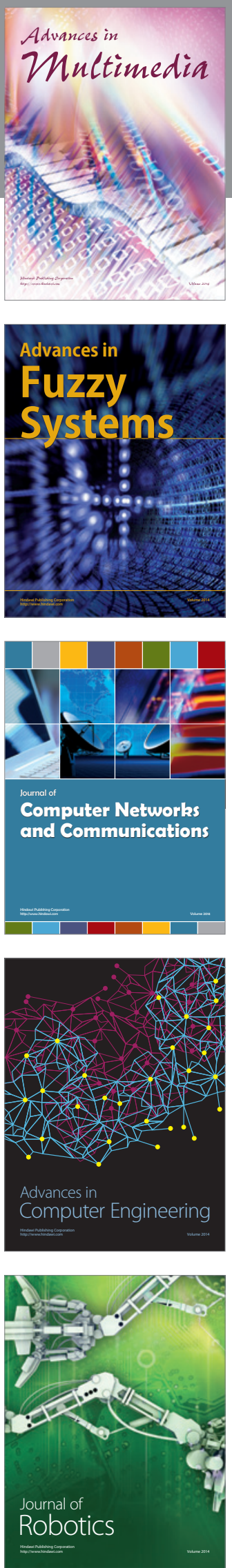

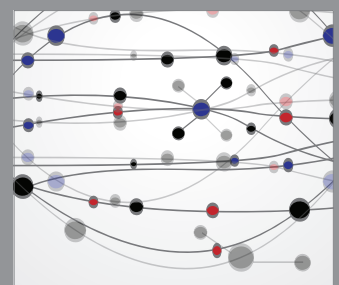

The Scientific World Journal
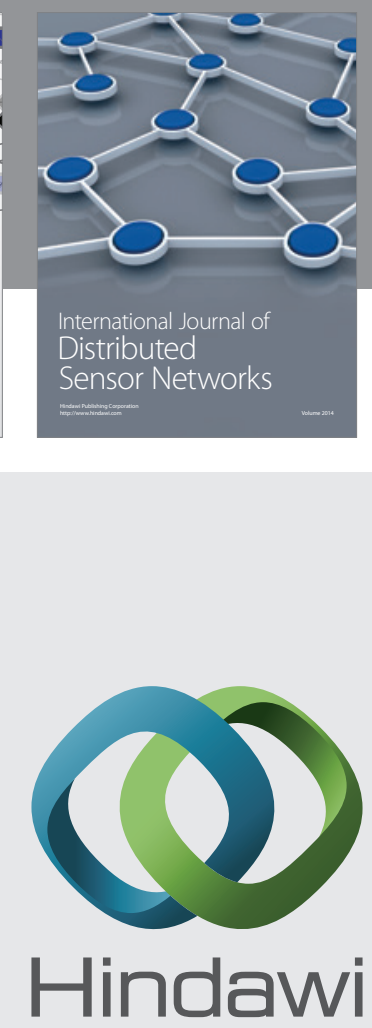

Submit your manuscripts at

http://www.hindawi.com
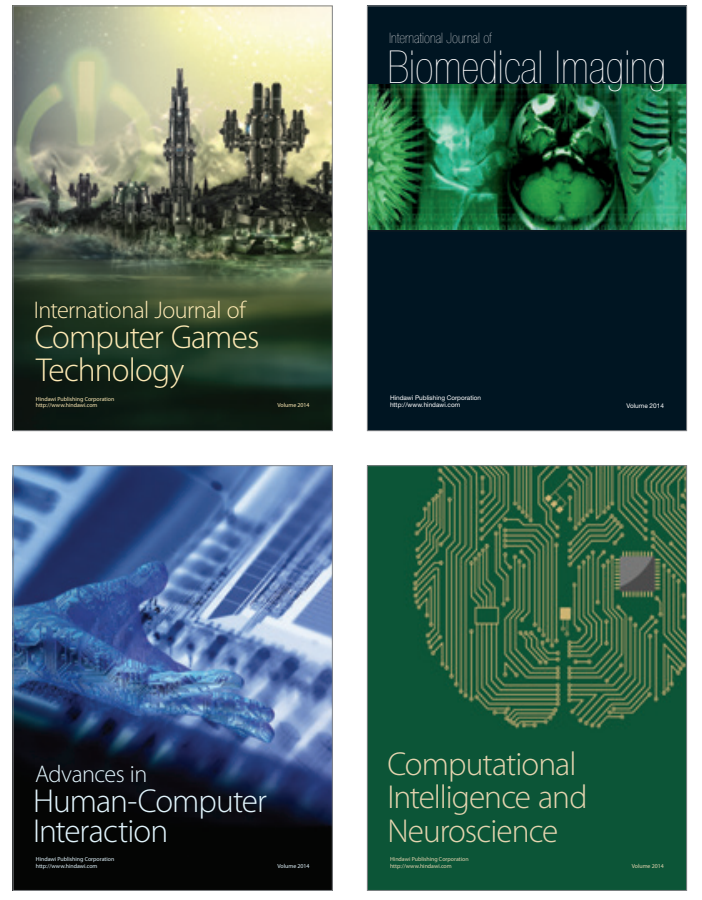
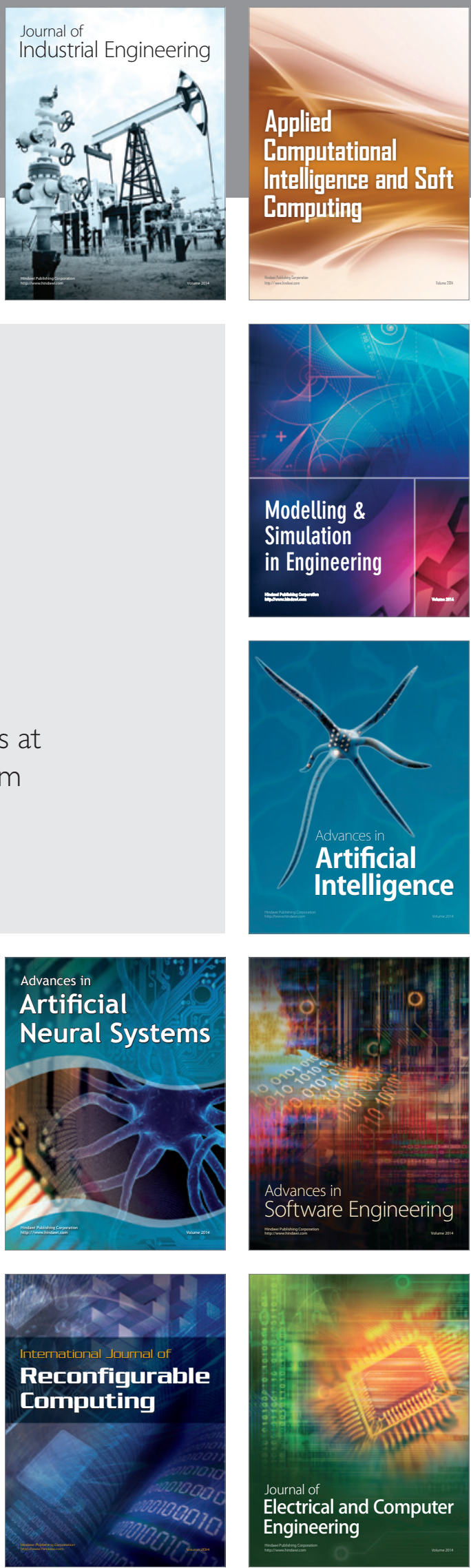